\title{
ARQUEOMINERALOGÍA DE CUENTAS DE LOS VALLES ORIENTALES DEL NORTE DE SALTA, ARGENTINA
}

\author{
ARCHAEOMINERALOGY OF BEADS FROM THE EASTERN VALLEYS \\ OF NORTHERN SALTA, ARGENTINA
}

María Florencia Becerra a, Beatriz N. Ventura ${ }^{B}$, Patricia Solác, Mariana Rosenbusch ${ }^{D}$, Guillermo Cozzi ${ }^{\text {E }}$ Andrea Romano $^{\mathrm{F}}$

Se presenta la caracterización de 1088 cuentas provenientes de colecciones de museos y recolecciones de campo del norte de Salta, Argentina. Se identificaron cuentas confeccionadas sobre toba, pizarra, vidrio, material malacológico y minerales. Para 27 de ellas se usó MEB-EDX y DRX, registrándose turquesa, sodalita y ópalo, y se modificaron sus clasificaciones previas a partir de datos geoquímicos y observación macroscópica. Finalmente, se evalúan potenciales fuentes de aprovisionamiento líticas, malacológicas y alóctonas.

Palabras clave: Arqueomineralogía, Cuentas, Valles orientales, Salta.

This paper presents the characterization of 1088 beads from museum collections and field collections from the eastern valleys of northern Salta, Argentina. Beads made of tuff, slate, glass, malacological material, and minerals were identified. 27 beads were analyzed through SEM-EDX and XRD revealing turquoise, sodalite and opal, and their previous classifications were modified from geochemical data and macroscopic observation. Finally, potential lithic, malacological, and foreign supply sources were assessed.

Keywords: Archaeomineralogy, Beads, Eastern Valleys, Salta.
En el mundo andino, ciertos bienes ornamentales fueron muy requeridos, entre ellos, las cuentas manufacturadas con rocas y minerales, sobre material malacológico, en metales y hueso. Estas piezas tuvieron valor como adornos personales, siendo también aplicadas en tabletas de madera, máscaras y vasos. Además, se depositaron como ofrendas funerarias y en las abras de las montañas junto a mineral molido, ritualidad vinculada con el culto a los cerros y los volcanes, con el tránsito de larga distancia y prácticas de pastores-mineros (Berenguer 2004, Nielsen et al. 2017). El pequeño tamaño de estas piezas y su durabilidad les otorgaron gran potencial para su circulación e intercambio (Soto 2019).

El color fue uno de los principales atributos considerados en la selección de las materias primas, así como el brillo y textura del mineral, su facilidad para ser trabajado y su abundancia. Pudieron influir también en su valoración la distancia o la dificultad para adquirir el material, su carácter "exótico".

A María Florencia Becerra, Universidad de Buenos Aires, Facultad de Filosofía y Letras, Instituto de Arqueología, Buenos Aires, Argentina. Consejo Nacional de Investigaciones Científicas y Técnicas (CONICET), Buenos Aires, Argentina. OrCID: 0000-0001-6302-7452. E-mail: florenciabecerra@gmail.com

B Beatriz N. Ventura, Universidad de Buenos Aires, Facultad de Filosofía y Letras, Instituto de Arqueología, Buenos Aires, Argentina. Consejo Nacional de Investigaciones Científicas y Técnicas (CONICET), Buenos Aires, Argentina. Orcid: 0000-0002-6834-2053. E-mail: beatrizventura2006@yahoo.com.ar

c Patricia Solá, Universidad de Buenos Aires, Facultad de Filosofía y Letras, Instituto de Arqueología, Buenos Aires, Argentina. Consejo Nacional de Investigaciones Científicas y Técnicas (conicet), Buenos Aires, Argentina. Orcid: 0000-0002-3608-7967. E-mail: patriciasola@gmail.com

D Mariana Rosenbusch, CONICET, Centro Atómico Constituyente (CAC), Buenos Aires, Argentina. OrCID: 0000-0001-6988-1043. E-mail: rosenbus@cnea.gov.ar

E Guillermo Cozzi, Intemin-segemar, Buenos Aires, Argentina. Orcid: 0000-0003-1909-955. E-mail: guillermo.cozzi@segemar.gov.ar

F Andrea Romano, InTEMin-SEgemar, Buenos Aires, Argentina. Orcid: 0000-0002-5035-7345. E-mail: andrea.romano@segemar.gov.ar 
Durante el Tawantinsuyu, los adornos del Inca y de la nobleza consistían en grandes tocados, pectorales, orejeras, coronas, brazaletes de metales preciosos y collares confeccionados con piedras semipreciosas -como la turquesa- o con materiales de profunda significación -como el mullu (Spondylus sp.)-. El valor simbólico de ciertos elementos o materiales y los ritos en que se utilizaban los hizo necesarios en la vida social y religiosa. Por esta razón, la actividad minera para fines metalúrgicos y manufactura lapidaria fue estimulada y controlada por el Estado (Berthelot 1978, Cantarutti 2013, Salazar et al. 2013), aunque también pudo efectuarse fuera del ámbito imperial (Garrido 2017).

El hallazgo de cuentas manufacturadas con diversos materiales se ha registrado en sitios arqueológicos de distintas cronologías en el Noroeste Argentino (NOA), pero en pocos casos se hicieron análisis específicos de sus materias primas. Generalmente, la identificación fue únicamente macroscópica, lo cual muchas veces confundió especímenes minerales de coloración similar. ${ }^{1}$ La sola distinción cromática ha llevado a clasificar, por ejemplo, a la sodalita como lapislázuli o a la turquesa como malaquita, subestimando la variabilidad mineralógica y geoquímica de los minerales empleados en determinada región. Asimismo, desde la arqueología no se ha logrado aún un estudio cabal de la literatura geológica sobre posibles fuentes de extracción de minerales para manufacturas lapidarias en el NOA.

En diversos asentamientos de los valles orientales del norte de Salta, Argentina, se ha recuperado una gran cantidad de cuentas, la mayoría de ellas confeccionadas con minerales de color verde, turquesa y azul y, en menor proporción, líticas, malacológicas y de vidrio. Como parte de los estudios de colecciones y de campo que hemos realizado en esta región, presentamos aquí una caracterización de las mismas, evaluando materia prima, cantidad, forma, color y su potencial confección in situ (presencia de preformas y accesibilidad a materias primas). Completamos el análisis con el estudio geoquímico de 27 cuentas minerales y una lítica, desde una visión analítica sustentada en la arqueomineralogía. Esta subdisciplina de la arqueometría permite caracterizar y clasificar materiales, analizar procesos posdepositacionales y plantear posibles fuentes de aprovisionamiento (Rapp 2009). Sobre la base de los resultados obtenidos, y a partir del análisis de bibliografía especializada, mencionamos las principales fuentes de los minerales detectados en la muestra, principalmente en los actuales territorios de Argentina, Chile, Bolivia y Perú.

\section{LOS VALLES ORIENTALES DEL NORTE DE SALTA}

Nuestros trabajos arqueológicos se realizan en los valles de Iruya, Nazareno y Bacoya, en el norte de Salta (Argentina), ubicados en la ladera oriental de las serranías de Santa Victoria (cordillera Oriental) (fig. 1). Este sector limita al oeste con la Puna de Jujuy y, al este, con los bosques montanos y la selva de las Yungas salteñas. Abarca desde los 4000 msnm en las serranías hasta los $2000 \mathrm{msnm}$ en los valles de los ríos. La intensa disección fluvial ha generado un relieve escarpado con pendientes pronunciadas y quebradas profundas. En la franja de los 2700-3300 msnm, se extienden los pastizales de Neblina (Brown \& Grau 1993), que durante la estación lluviosa (verano) se transforman en verdes praderas. Allí, gracias a las condiciones microclimáticas y edafológicas, se han emplazado los asentamientos humanos, antiguos y actuales, y se ha construido una extensa andenería agrícola en las terrazas fluviales y en las laderas de los cerros.

\section{Geología}

La estructura de la serranía de Santa Victoria se debe a una tectónica esencialmente vertical ("tectónica de bloques") que elevó el basamento precámbrico conservando una configuración longitudinal de rumbo dominante NNEsso. Las rocas más antiguas son las metasedimentitas plegadas de la Formación Puncoviscana representadas por pizarras, filitas, cuarcitas y metagrauvacas. En forma paralela al rumbo predominante y en discordancia sobre el basamento prepaleozoico, se disponen potentes secuencias estratigráficas de conglomerados y ortocuarcitas (Cámbrico), seguidas de lutitas, fangolitas y cuarcitas (Ordovícico), areniscas, limoarcilitas, conglomerados y calizas (Cretácico), y areniscas, limolitas y arcilitas margosas (Paleógeno). Los materiales cuaternarios son escasos depósitos aluvio-coluviales, lacustres, palustres y fluvioglaciares emplazados en los flancos de las quebradas y formando conos de deyección. La secuencia culmina con ocasionales tobas preservadas en la porción cuspidal de algunos cerros, a menos de $10 \mathrm{~km}$ de las 


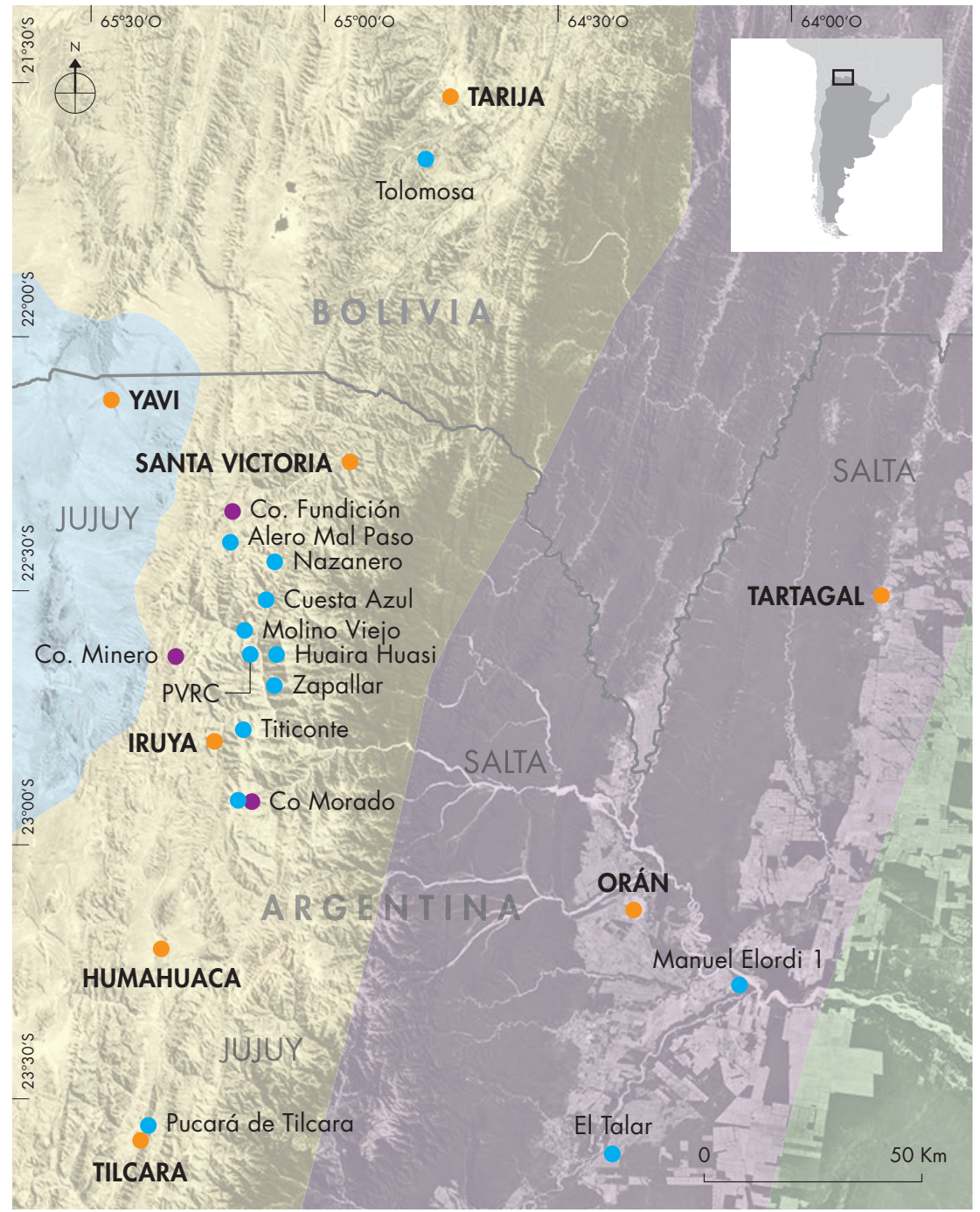

\begin{tabular}{|l|}
\hline SIMBOLOGía \\
- Sitio arqueológico \\
- Localidad \\
Cerro \\
Puna de Jujuy y \\
Altiplano boliviano \\
Cordillera Oriental \\
(Argentina y Bolivia) \\
Sierras Subandinas \\
(Argentina) y Frente \\
Subandino (Bolivia) \\
Llanura chaqueña \\
\hline
\end{tabular}

Figura 1. Mapa del área de estudio con los sitios arqueológicos mencionados. Figure 1. Map of the study area with the archaeological sites mentioned in the text. localidades de Iruya y Rodeo Colorado. Los recursos minerales del área incluyen filones polimetálicos con cobre, plomo, zinc, níquel, uranio y baritina, y placeres auríferos (Rubiolo et al. 2003).

\section{Arqueología}

Las primeras excavaciones arqueológicas en estos valles se realizaron en el santuario de altura de Cerro Morado por Eduardo Casanova (1930) y en Titiconte por Salvador Debenedetti y Eduardo Casanova (1933-1935) durante la xxv Expedición Arqueológica de la Facultad de Filosofía y Letras de la Universidad de Buenos Aires (xxve). Fernando Márquez Miranda (1939) excavó también en
Titiconte y en algunos de los diversos poblados que registró en el área, recuperando numerosas piezas que integran la Colección Márquez Miranda (смм) del Museo de La Plata (MLP) de la Universidad Nacional de La Plata. ${ }^{2}$ Titiconte es considerado un centro administrativo inca, rodeado por una importante andenería agrícola (Raffino et al. 1986). Presenta una compleja arquitectura con recintos rectangulares y grandes recintos subterráneos circulares, techos en falsa bóveda y pisos cubiertos con lajas, con vanos trapezoidales en las aberturas y nichos en las paredes. Por el contrario, los otros sitios de la región que habrían estado habitados durante la ocupación inca son asentamientos residenciales conformados por 10 a 30 recintos de formas 
circulares-elípticas, de 4 a $7 \mathrm{~m}$ de diámetro, con paredes dobles y, en algunos casos, uso de piedras canteadas. En las cercanías hay extensos andenes de cultivo. Una excepción por su mayor dimensión es Pueblo Viejo de Rodeo Colorado (PVRC) con unos 100 recintos de formas circulares-elípticas y muros dobles. Este sitio presenta formas de entierro similares a las detectadas en Titiconte y materiales asignables a momentos incaicos, aunque registra también ocupaciones previas al $1000 \mathrm{AD}$ y preincaicas. Hasta el momento, solo se han fechado las ocupaciones más antiguas del sitio.

El estudio de las mencionadas colecciones arqueológicas, los nuevos trabajos de campo, el análisis de documentación, de cartografía histórica y de bibliografía geológica permite plantear la posibilidad del desarrollo de actividades minero-metalúrgicas en los yacimientos de cobre, plata y oro de la región por parte del Estado inca (Ventura \& Scambato 2013, Ventura \& Oliveto 2014). Para ello, se habrían trasladado poblaciones y grupos jerarquizados especializados en minería, metalurgia, tareas administrativas y de control. El Memorial de Ledesma Valderrama de 1631 menciona que estos valles orientales estaban ocupados por "dos naciones de indios naturales del Perú", una de churumatas y la otra de "ingas del Cusco", y ambas son "gente rica de plata y minerales" (Ventura \& Oliveto 2014). Junto con estas actividades extractivas se habría generado un fuerte desarrollo agrícola, con grandes obras de andenería y almacenaje para abastecer a los trabajadores, y la instalación de Titiconte como centro administrativo y de un santuario de altura en Cerro Morado (Casanova 1930, Raffino et al. 1986).

En este contexto, cobran relevancia los numerosos elementos suntuarios recuperados en los sitios PVRC, Molino Viejo, Huaira Huasi, Zapallar y Titiconte. Se trata de piezas de metal, adornos y posibles amuletos hallados mayormente en entierros en el interior de recintos habitacionales (Márquez Miranda 1939). Algunas de estas piezas -de oro, plata y bronce (tincurpas, chipanas)han sido consideradas insignias y adornos de jerarquía de la sociedad inca (Horta Tricallotis 2008) y de valor ritual en ámbitos mineros (bolsas conteniendo mineral, instrumentos musicales, bola de plomo) (González \& Westfall 2008, Brooks et al. 2013). Además, en dichos sitios, se registraron abundantes cuentas, cuyas materias primas fueron catalogadas, sin mayores análisis, como malaquita, lapislázuli, barro cocido, valvas de molusco y nácar. Las mismas son el objeto de esta investigación.

\section{MATERIALES Y MÉTODOS}

La muestra registrada comprende cuentas de las colecciones Márquez Miranda (CMM-MLP), de la Xxv Expedición depositada en el Museo Etnográfico Juan B. Ambrosetti, Buenos Aires (xxvE-ME) y de una colección privada reunida por un poblador de la zona. También incluye cuentas recuperadas durante las campañas de 2013 y 2016 (tabla 1), sumando un total de 1088 cuentas.

Las cuentas de la CMM-MLP están organizadas en 77 lotes que tienen entre 1 y 129 cuentas. De estos, 10 no fueron localizados en el depósito, por lo que el total de cuentas estudiadas corresponde únicamente a 67 lotes (994 cuentas), provenientes de PVRC, Molino Viejo, Cuesta Azul, Titiconte, Huaira Huasi y Zapallar. ${ }^{3}$

La colección XXVE-ME está conformada por cuentas recuperadas en Titiconte y Cerro Morado, aunque la cantidad de piezas en el museo (61) es menor que la informada en las publicaciones (100 "cuentas de malaquita y lapislázuli” en Titiconte y 30 en Cerro Morado) (Casanova 1930, Debenedetti \& Casanova 1933-1935).

La colección privada consta de 21 cuentas halladas en superficie en PVRC. Las muestras recuperadas en nuestros trabajos de campo provienen de este sitio y de Alero Mal Paso. La mayoría son de recolección superficial excepto tres de PVRC: una hallada en la excavación de un recinto habitacional (estructura 7) $\mathrm{y}$ dos dentro de una vasija funeraria registrada en un rescate arqueológico.

La muestra analizada comprende cuentas manufacturadas sobre diversas materias primas clasificadas como minerales $(\mathrm{N}=783)$, líticas $(\mathrm{N}=201)$, malacológicas $(\mathrm{N}=57)$, de vidrio $(\mathrm{N}=4)$ y no determinadas $(\mathrm{N}=43)$. En el caso de la CMM-MLP, casi la mitad de los lotes agrupan cuentas de aparente similar composición; el resto combina cuentas líticas, minerales de distintos colores y malacológicas provenientes, probablemente, de un mismo hallazgo. Respecto de los lotes, 39 tienen entre 1 y 5 cuentas, 14 lotes tienen entre 6 y 20 cuentas; otros 14 alcanzan más de 20 piezas, y de ellos, 5 lotes tienen entre 50 y 129 cuentas. Si consideramos que cada lote proviene de un contexto funerario distinto y que estas piezas han sido adornos (collares, pulseras, colgantes), la mayoría de ellos tendría pocas cuentas. También podrían tratarse de ofrendas de cuentas sueltas, individuales $(\mathrm{N}=20)$ o de conjuntos muy pequeños, tal como las dos obtenidas del rescate realizado en PVRC (PVRC25 y 26). 
Tabla 1. Cuentas clasificadas por materia prima y color. N/D=No determinadas. Table 1. Beads classified according to raw material and color. $N / D=$ Undetermined.

\begin{tabular}{|c|c|c|c|c|c|c|c|c|c|c|c|}
\hline \multirow{3}{*}{$\begin{array}{l}\text { zó } \\
0 \\
0 \\
0 \\
0 \\
0\end{array}$} & \multirow{3}{*}{$\stackrel{\circ}{\circ}$} & \multirow{3}{*}{ 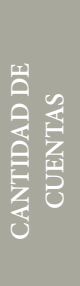 } & \multirow{3}{*}{ 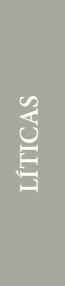 } & \multicolumn{5}{|c|}{ MINERALES } & \multirow{3}{*}{ 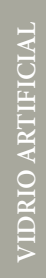 } & \multirow{3}{*}{ 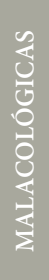 } & \multirow{3}{*}{$\frac{\theta}{z}$} \\
\hline & & & & \multicolumn{3}{|c|}{ VERDES } & \multirow[b]{2}{*}{ 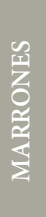 } & \multirow[b]{2}{*}{ 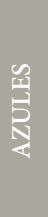 } & & & \\
\hline & & & & 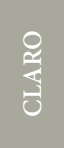 & 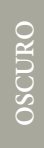 & 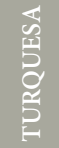 & & & & & \\
\hline \multirow{7}{*}{ СMM } & PVRC & 937 & 197 & 164 & 29 & 430 & 11 & 25 & - & 47 & 34 \\
\hline & Molino Viejo & 37 & 2 & 5 & 2 & 23 & - & 1 & - & 2 & 2 \\
\hline & Titiconte & 6 & - & - & - & 1 & - & 5 & - & - & - \\
\hline & Zapallar & 1 & - & - & - & - & 1 & - & - & - & - \\
\hline & Huaira Huasi & 3 & 1 & - & - & - & - & - & - & - & 2 \\
\hline & Cuesta Azul & 10 & - & 1 & - & 2 & - & - & 4 & - & 3 \\
\hline & Subtotal & 994 & 200 & 170 & 31 & 456 & 12 & 31 & 4 & 49 & 41 \\
\hline \multirow{3}{*}{ XXVE } & Titiconte & 49 & - & 10 & 8 & 24 & - & 6 & - & - & 1 \\
\hline & Cerro Morado & 12 & - & 3 & - & 6 & - & 1 & - & 1 & 1 \\
\hline & Subtotal & 61 & - & 13 & 8 & 30 & - & 7 & - & 1 & 2 \\
\hline \multirow{2}{*}{$\begin{array}{l}\text { Col. } \\
\text { priv. }\end{array}$} & PVRC & 21 & 1 & 3 & - & 3 & - & 13 & - & 1 & - \\
\hline & Subtotal & 21 & 1 & 3 & - & 3 & - & 13 & - & 1 & - \\
\hline \multirow{4}{*}{$\begin{array}{c}\text { Recolec. } \\
\text { campo }\end{array}$} & PVRC & 6 & - & 2 & - & 2 & - & 2 & - & - & - \\
\hline & Alero Mal Paso & 6 & - & - & - & - & - & - & - & 6 & - \\
\hline & Subtotal & 12 & - & 2 & - & 2 & - & 2 & - & 6 & - \\
\hline & Total & 1088 & 201 & 188 & 39 & 491 & 12 & 53 & 4 & 57 & 43 \\
\hline
\end{tabular}

Al iniciar este estudio, contábamos con la identificación de dos cuentas de toba y una malacológica, e información mineralógica del análisis previo realizado sobre seis muestras de la CMM-MLP mediante difracción de rayos X: dos cuentas de color verde (claro y oscuro) determinadas como turquesa, una cuenta azul verdosa identificada como ópalo, una de vidrio y dos cuentas azules de sodalita, una de ellas con una costra de taranakita (Ventura \& Scambato 2013).

Para este trabajo, la estrategia seguida consistió en agrupar las cuentas minerales en conjuntos de posible similitud composicional de acuerdo con características macroscópicas, especialmente el color. Aunque es una propiedad poco diagnóstica, resultó operativa para abordar la cantidad de piezas de la muestra total, considerando que el análisis físico-químico de muestras de cada conjunto permitiría confirmar si se trataba de un mismo mineral o si habíamos agrupado cuentas de diferentes especies minerales. Se formaron así conjuntos de color turquesa (tonalidades azul claro y oscuro, y verdes), verde claro y verde oscuro, azules y marrones (a veces, parcialmente moteadas y con bandas verdes y/o azules). Luego, se seleccionaron 20 muestras de la CMM-MLP ${ }^{4}$ (19 representativas de cada conjunto de color y una lítica con venilla verde), una cuenta azul de la colección privada de PVRC y seis recuperadas por nosotros en este sitio $\left(\mathrm{N}_{\mathrm{T}}=27\right)$ (tabla 2). 
Las cuentas minerales seleccionadas fueron estudiadas mediante dos técnicas analíticas instrumentales complementarias: 1) espectrometría de rayos $\mathrm{X}$ por dispersión de energía incorporada a un microscopio electrónico de barrido y 2) difracción de rayos $\mathrm{X}$ por polvo.

La espectrometría de rayos $\mathrm{X}$ por dispersión de energía (EDX) permite el análisis cualitativo y semicuantitativo de la superficie de muestras sólidas sin necesidad de producir la destrucción o alteración de estas, cumpliendo con uno de los objetivos primordiales en la práctica analítica de muestras museísticas que es la preservación de los materiales. Por motivos de disponibilidad, se utilizaron dos equipos MEB-EDX, un microscopio electrónico de barrido ambiental Philips XL 30 ESEM con microsonda dispersiva en energía marca EDAX del INTEMIN-SEGEMAR y un microscopio electrónico de barrido Philips 515 provisto de un analizador EDAX del Centro Atómico Constituyentes (CAC), ambos en Buenos Aires.

Los resultados de EDX presentados en la tabla composicional son el promedio de un mínimo de tres mediciones puntuales en distintas zonas de las muestras, considerando para la cuantificación los elementos detectados a partir del sodio $(\mathrm{Na})$.

La difracción de rayos X (DRX) por polvo permite identificar y cuantificar fases cristalinas, en particular minerales, que requieren preferentemente de una preparación previa de la muestra. Esta incluye la micronización por pulverizado y la disposición al azar de las partículas con el propósito de obtener un difractograma experimental que pueda ser comparado con los patrones de difracción de cada fase. Dado que, por ser materiales de colección no podían ser pulverizados, se seleccionaron para el estudio solo aquellas cuentas que presentaban una superficie plana y un espesor menor a $5 \mathrm{~mm}$ y que podían ser montadas en portamuestras especiales. Para la interpretación de los difractogramas se tuvo en cuenta el tipo de montaje especificado previendo que, en función de la orientación preferencial y del tamaño de grano de algunas de las fases cristalinas presentes en las cuentas, se producirían variaciones en la intensidad relativa e incluso, posiblemente, la extinción de algunos picos.

La colección de datos se realizó con un difractómetro de rayos $\mathrm{X}$ Philips, modelo $\mathrm{X}^{\prime}$ Pert $\mathrm{PRO}$, con radiación $\mathrm{Ka}$ de $\mathrm{Cu}$, en intervalos de barrido 2 theta de 4 a $70^{\circ}$ con tamaño de paso/tiempo de $0,03^{\circ} / 2$ segundos. El análisis de datos se llevó a cabo utilizando el programa
Tabla 2. Descripción de la muestra analizada. * Análisis DRX previos realizados por Ventura. ${ }^{* *}$ Análisis DRX realizados sobre polvo mineral. Table 2. Description of the sample analyzed. ${ }^{*}$ Previous DRX analyses carried out by Ventura. ${ }^{* *}$ DRX analyses on mineral dust.

\begin{tabular}{|c|c|c|}
\hline ID & LOTE & COLOR \\
\hline PVRC1 $^{*}$ & $7291-24872$ & Azul \\
\hline PVRC $^{*}$ & $7293-24934-25058$ & Azul (con costra marrón) \\
\hline PVRC7 & $7933-26163$ & Azul-marrón \\
\hline PVRC19 & $7934-26165 / 382$ & Azul \\
\hline PVRC20 & $7934-26165 / 382$ & Azul (con costra marrón) \\
\hline $\operatorname{PVRC} 27^{* *}$ & Superficie & Azul \\
\hline PVRC28 & Colección privada & Azul \\
\hline PVRC29 & E7-Cuad1-C2 & Azul \\
\hline Mv200* & $8016-26284$ & Verde claro \\
\hline PVRC9 & $7286-24628$ a 24683 & Verde claro \\
\hline PVRC10 & $7294-25059$ a 25181 & Verde claro \\
\hline PVRC14 & $7920-26150 / 322$ & Verde claro \\
\hline PVRC15 & $7920-26150 / 322$ & Verde claro \\
\hline PVRC16 & $7281-24583$ a 24589 & Verde claro \\
\hline PVRC25 & Rescate & Verde claro \\
\hline PVRC26 & Rescate & Verde claro \\
\hline PVRC18 & $8012-26280$ & Verde oscuro \\
\hline MV201 & $8014-26282$ & Verde oscuro \\
\hline PVRC2* $^{*}$ & $7933-26163$ & Verde oscuro \\
\hline PVRC8 & $7933-26163$ & Turquesa \\
\hline PVRC12 & $7294-25059$ a 25181 & Turquesa \\
\hline PVRC13 & $7920-26150 / 322$ & Turquesa \\
\hline PVRC17 & $8012-26280$ & Turquesa \\
\hline PVRC23 & $7890-26118$ & Turquesa \\
\hline PVRC $24^{* *}$ & Sup. E31 y M34 & Turquesa \\
\hline PVRC $30^{* *}$ & Sup. OE cista 2 & Turquesa \\
\hline PVRC5 & $7995-26262$ & Lítica con venilla verde \\
\hline PVRC6 & $7932-26162$ & Marrón \\
\hline PVRC11 & $7294-25059$ a 25181 & Verde oscuro-marrón \\
\hline PVRC22 & $7890-26118$ & Azul-verde-marrón \\
\hline $\mathrm{Z} 100^{*}$ & $7554-25782$ & Turquesa \\
\hline PVRC21 & $7928-26158$ & Azul claro \\
\hline
\end{tabular}


High Score Plus de PANalytical y la base de datos PDF del ICDD (1997). Los estudios por difracción se hicieron en Ciga-Intemin-Segemar, Parque Tecnológico Miguelete, Buenos Aires.

Las 27 muestras seleccionadas se analizaron mediante SEM-EDX y se compararon los resultados con las fórmulas estequiométricas de turquesa y sodalita, dos de las especies minerales determinadas previamente por DRX. Las difracciones se efectuaron directamente sobre las cuentas, salvo tres muestras (recolección de campo 2013), de las cuales se extrajeron diminutos fragmentos para su pulverización. El tamaño de las cuentas PVRC5 Y PVRC2O (CMM-MLP) imposibilitó su análisis por DRX.

Las cuentas malacológicas de Alero Mal Paso y Cerro Morado (ME) $(\mathrm{N}=7)$ fueron identificadas por Sergio Miquel (CONICET/Museo de Ciencias Naturales B. Rivadavia, Buenos Aires).

\section{LAS CUENTAS}

\section{Cuentas minerales}

Son las más numerosas $(\mathrm{N}=783)$ y fueron agrupadas por color en: 718 verdes (491 de diversas tonalidades turquesas, 188 verde claro y 39 verde oscuro), 53 azules y 12 marrones (con bandas o motas verdes y/o azules). Veinte cuentas azules de la CMM-MLP presentan una costra irregular blanquecina a castaña.

Los análisis físico-químicos permitieron caracterizar esos conjuntos preliminares, confirmando dicha agrupación. Las composiciones elementales determinadas por EDX en 16 cuentas de PVRC y Molino Viejo, seleccionadas por sus tonalidades verde claro, verde oscuro y turquesa, muestran niveles elevados de aluminio (Al), fósforo $(\mathrm{P})$ y cobre $(\mathrm{Cu})$ en la proporción característica en que se encuentran constituyendo el mineral turquesa. El hierro (III), un elemento sustituyente habitual del Al en dicho mineral, está también presente en proporciones variables en estas muestras. Es factible que el resto de los elementos determinados en este grupo-Mg, Si, S, Ca y $\mathrm{K}-$, en concentraciones bajas a intermedias, provenga de otros minerales presentes en aquellas cuentas que, a simple vista, exhiben heterogeneidades cromáticas. El análisis DRX confirmó, en todos los casos, la existencia de turquesa asociada ocasionalmente con otros minerales como cuarzo, feldespato, lazurita y apatita (tabla 3 y fig. 2).
También se realizaron análisis EDx en seis cuentas azules de PVRC. Tres arrojaron altos porcentajes de $\mathrm{Na}$, $\mathrm{Al}, \mathrm{Si} \mathrm{y} \mathrm{Cl}$, elementos característicos del mineral sodalita, aunque en proporciones no perfectamente concordantes con la composición estequiométrica del mineral; sin embargo, el mineral fue identificado por DRX en las cinco muestras analizadas por esta técnica (PVRC7, 19, 27, 28 y 29). En todas estas cuentas se registraron bajas concentraciones de $\mathrm{Mg}, \mathrm{K}$ y Ti, y bajas a intermedias de Ca y Fe; así como variable contenido de P, siendo PVRC7 y PVRC28 las que tienen elevado porcentaje de este elemento. Los porcentajes de P determinados indican la presencia de un fosfato.

El tamaño de la cuenta PVRC20 imposibilitó su análisis mediante DRX. Presentaba una costra blanquecinaamarronada, similar a la de la muestra PVRC4 analizada previamente por Ventura, cuyos resultados DRx habían indicado sodalita y taranakita. La composición elemental de la costra (sobre el mineral azul de base) determinada por EDX sería compatible con taranakita. Las otras cinco cuentas azules no contenían la mencionada costra; sin embargo, las composiciones elementales medidas, además de ser compatibles con sodalita, denotan la presencia de otro mineral, no detectado por DRX, cuyo elemento dominante es fósforo. Estas indefiniciones podrían deberse a la imposibilidad de pulverización de las muestras para su análisis, siendo PVRC27 la única cuenta azul analizada mediante DRX por polvo en esta etapa (tabla 3 y fig. 3 ).

El resto de las cuentas minerales analizadas consiste en una marrón (PVRC6), una azul claro (PVRC21), una verde oscuro-marrón (PVRC11) y una azul-verde-marrón (PVRC22). Los análisis EDX arrojaron valores muy elevados de $\mathrm{Si}$, indicativos de la presencia masiva de un compuesto silíceo, identificado por DRX como ópalo en las muestras PVRC6, PVRC11 y PVRC22. La muestra PVRC21 contiene elevado Si y Cu. Su difractograma de baja intensidad no permitió determinar la especie mineral, aunque su composición es compatible con crisocola (tabla 3 y fig. 4).

Las formas de las cuentas son variadas. Del conjunto de cuentas azules, 32 son tubulares $\left(\mathrm{N}_{\mathrm{T}}=53\right)$, y 657 del conjunto de color turquesa azul/verde $\left(\mathrm{N}_{\mathrm{T}}=718\right)$ y 9 marrones $\left(\mathrm{N}_{\mathrm{T}}=11\right)$ son circulares planas. Este tipo de cuentas presentan diámetros entre 1,5 y $23 \mathrm{~mm}$, aunque la mayoría no supera los $10 \mathrm{~mm}$, y las tubulares tienen largos comprendidos entre 4 y $29 \mathrm{~mm}$. En la CMM-MLP registramos tres cuentas planas verdes de mayor tamaño, 
Tabla 3. Análisis de las cuentas por EDX y DRX. * Análisis DRX previos realizados por Ventura. ${ }^{\star \star}$ Análisis DRX sobre polvo mineral. (?) Componente de identificación dudosa. Ópalo-CT: ópalo (cristobalita-tridimita). Table 3. EDX and DRX beads analyses. ${ }^{*}$ Previous DRX analyses carried out by Ventura. ${ }^{* *}$ DRX analyses on mineral dust. (?) Component of uncertain identification. Ópalo-CT: opal (cristobalite-tridimite).

\begin{tabular}{|c|c|c|c|c|c|c|c|c|c|c|c|c|c|}
\hline \multirow[t]{2}{*}{ ID } & \multicolumn{12}{|c|}{ EDX (\% en peso) } & \multirow[t]{2}{*}{ DRX } \\
\hline & $\mathrm{Na}$ & $\mathrm{Mg}$ & $\mathrm{Al}$ & $\mathrm{Si}$ & $\mathrm{P}$ & s & $\mathrm{Cl}$ & $\mathrm{K}$ & $\mathrm{Ca}$ & $\mathrm{Ti}$ & $\mathrm{Fe}$ & $\mathrm{Cu}$ & \\
\hline PVRC1 $^{*}$ & - & - & - & - & - & - & - & - & - & - & - & - & Sodalita \\
\hline PVRC $4^{*}$ & - & - & - & - & - & - & - & - & - & - & - & - & Sodalita + taranakita + cuarzo \\
\hline PVRC7 & 1,6 & 1,2 & 32,6 & 3,9 & 31,6 & - & - & 2,8 & 12,5 & - & 13,8 & - & Sodalita \\
\hline PVRC19 & 23,3 & 0,8 & 25,7 & 34,2 & 0,8 & - & 8,9 & 2,9 & 2,7 & - & 0,6 & - & Sodalita \\
\hline PVRC20 & 1,7 & 1,5 & 16,2 & 45,9 & 4,4 & - & - & 16 & 9,6 & - & 4,6 & - & - \\
\hline $\operatorname{PVRC} 27^{* *}$ & 13,3 & 1,2 & 28,4 & 27,7 & 15,1 & - & 7,5 & 2,6 & 2,1 & 2 & - & - & Sodalita + cuarzo \\
\hline PVRC28 & 1,5 & 1,8 & 23,4 & 20,4 & 25,5 & - & 2 & 5,1 & 12,8 & 1,2 & 6,7 & - & Sodalita \\
\hline PVRC29 & 10,4 & - & 28,9 & 34,5 & 10,4 & - & 10 & 3,6 & 2,1 & - & - & - & Sodalita + cuarzo? \\
\hline MV $200^{*}$ & - & - & - & - & - & - & - & - & - & - & - & - & Turquesa \\
\hline PVRC9 & - & - & 29,2 & 11,7 & 25,9 & 3,2 & - & 7,1 & 2,2 & - & 7,2 & 13,4 & Turquesa + cuarzo \\
\hline PVRC10 & - & 0,8 & 31,8 & 21,3 & 22,7 & 0,7 & - & 2,9 & 2,3 & - & 4,9 & 12,8 & Turquesa + cuarzo \\
\hline PVRC14 & - & - & 29,7 & 26,7 & 20 & 0,3 & - & 1,9 & 5,4 & - & 2,8 & 13,2 & Turquesa + apatita? \\
\hline PVRC15 & - & 0,8 & 38,9 & 10,9 & 27 & - & - & 1,4 & 2,4 & - & 3,5 & 15,1 & Turquesa + cuarzo + feldespato? \\
\hline PVRC16 & - & 0,5 & 32,9 & 13 & 24,7 & - & - & 1,5 & 5,2 & - & 6,9 & 15,2 & Turquesa + apatita? \\
\hline PVRC25 & - & - & 32,5 & 14,5 & 32,9 & - & - & 1,7 & - & - & 4,4 & 13,9 & Turquesa + cuarzo \\
\hline PVRC26 & - & - & 31,9 & 16,1 & 33,7 & - & - & 1,4 & - & - & 4,1 & 12,9 & Turquesa \\
\hline PVRC18 & - & - & 42,9 & 3,6 & 30,1 & - & - & - & 1,1 & - & 1,9 & 20,5 & Turquesa \\
\hline MV201 & - & - & 41,1 & 5,7 & 28,4 & - & - & - & 1,2 & - & 2,8 & 20,9 & Turquesa + cuarzo \\
\hline PVRC2* & - & - & - & - & - & - & - & - & - & - & - & - & Turquesa \\
\hline PVRC8 & - & - & 43,8 & 1,7 & 30,8 & - & - & - & 2,2 & - & 1,6 & 19,9 & Turquesa + cuarzo + feldespato \\
\hline PVRC12 & - & - & 45,1 & 3 & 30,3 & - & - & - & - & - & 2,1 & 19,4 & Turquesa + cuarzo \\
\hline PVRC13 & - & - & 38,5 & 7,3 & 27,6 & - & - & - & 1,9 & - & 4,4 & 20,3 & Turquesa + cuarzo \\
\hline PVRC17 & - & - & 42,4 & 3,2 & 30,2 & - & - & - & - & - & 3,9 & 20,3 & Turquesa + cuarzo \\
\hline PVRC23 & - & 0,5 & 39,9 & 3,5 & 29,3 & 0,5 & - & 0,6 & 1,1 & - & 4,1 & 20,6 & Turquesa + cuarzo \\
\hline PVRC $24^{* *}$ & - & - & 34,7 & 5 & 37,4 & - & - & - & - & - & 10,5 & 12,4 & Turquesa + cuarzo \\
\hline PVRC $30^{* *}$ & - & - & 37,2 & 7,3 & 32,7 & - & - & - & - & - & 4,9 & 18,6 & Turquesa + cuarzo + lazurita \\
\hline PVRC5 & - & - & 1,1 & 8,6 & 7,2 & 15,3 & 1,1 & 1,5 & 20,5 & - & - & 44,7 & - \\
\hline PVRC6 & - & 6,6 & 8,5 & 76,2 & - & - & - & 1,4 & 5,5 & - & 1,8 & - & Ópalo? \\
\hline PVRC11 & - & 4,9 & 7,5 & 80,1 & 2,6 & - & - & 1,3 & 1,1 & - & 2,4 & - & Ópalo-ст \\
\hline PVRC22 & - & 4,3 & 7 & 79,9 & 1,5 & - & - & 1,3 & 2,2 & - & 3,8 & - & Ópalo-Ст + cuarzo + paligorskita? \\
\hline $\mathrm{Z} 100^{*}$ & - & - & - & - & - & - & - & - & - & - & - & - & Ópalo \\
\hline PVRC21 & - & 2,9 & 8,9 & 62,2 & - & - & - & 6,2 & 3 & - & - & 16,7 & Difractograma de baja intensidad \\
\hline
\end{tabular}




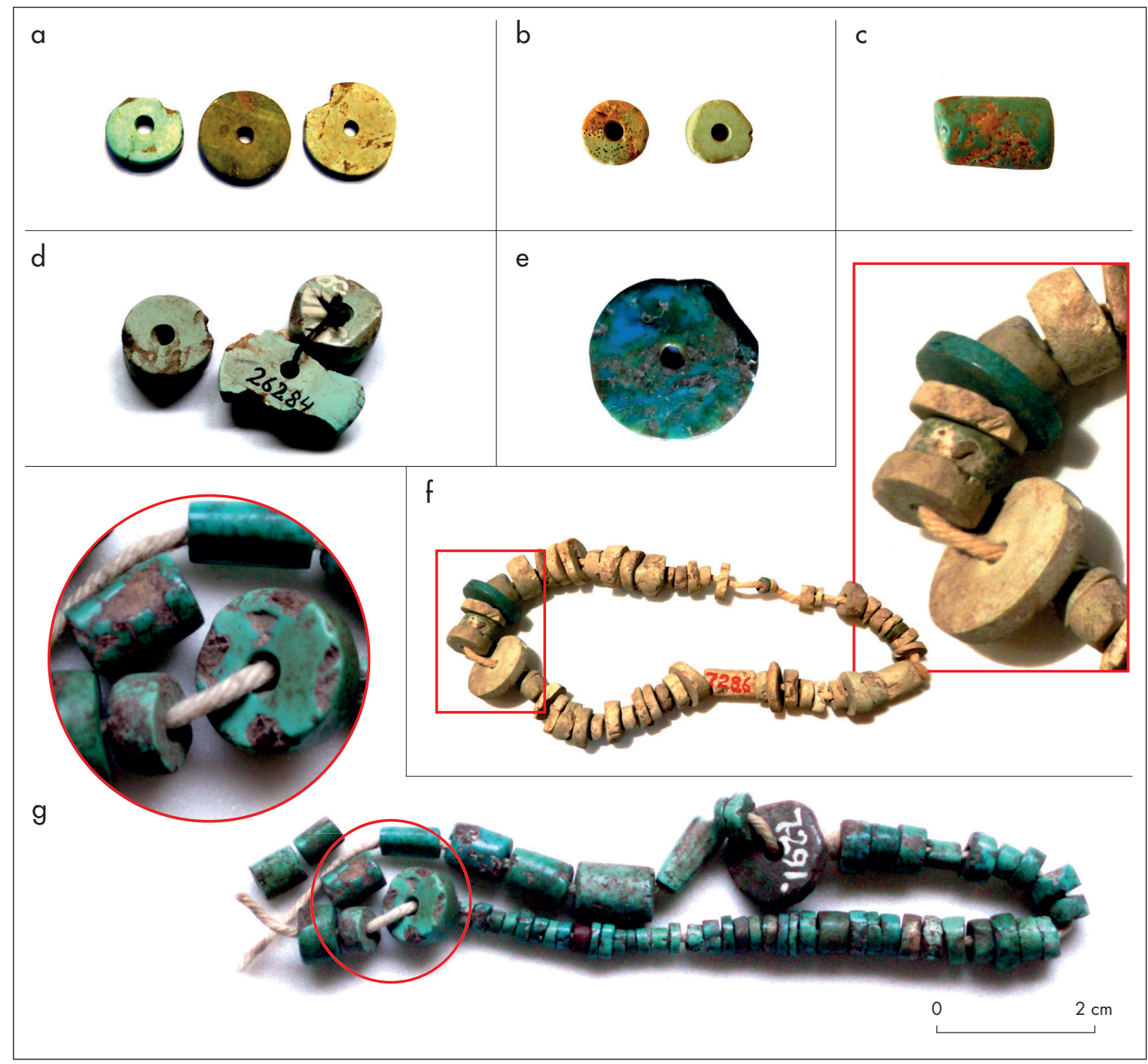

Figura 2. Cuentas confeccionadas con turquesa. El armado de los collares es actual: a) PVRC13, 14 y 15, MLP-Ar-CMM-7920-26150; b) PVRC25 y PVRC26, rescate arqueológico; c) PVRC24, recolección de campo; d) Molino Viejo-MV200, MLP-Ar-CMM-8016-26284; e) PVRC23, MLP-Ar-CMM-7890-26118; f) PVRC9, MLP-Ar-CMM-7286-24628 a 24683; g) PVRC, MLP-Ar-CMM-7291-24872 a 24932. Figure 2. Beads made of turquoise. The assembly of the necklaces is contemporary: $a$ ) PVRC13, 14 and 15, MLP-Ar-CMM-7920-26150; $\boldsymbol{b}$ ) PVRC25 and PVRC26, archaeological rescue; c) PVRC24, field collection; d) Molino Viejo-MV200, MLP-Ar-CMM-8016-26284; e) PVRC23, MLP-Ar-CMM-7890-26118; f) PVRC9, MLP-Ar-CMM-7286-24628 a 24683; g) PVRC, MLP-Ar-CMM-7291-24872 to 24932.

con formas ovalada, rectangular y de medialuna. No se hallaron preformas de cuentas minerales, aunque algunas piezas están parcialmente fragmentadas.

\section{Cuentas líticas}

Este conjunto ( $\mathrm{N}=201)$ está conformado principalmente por cuentas de toba. ${ }^{5}$ Unas pocas fueron identificadas como pizarra, lutita y probable caliza. La morfología de estas piezas es mayormente circular plana $(\mathrm{N}=156)$ con diámetros entre 5 y $45 \mathrm{~mm}$ y espesores entre 3 y 24 $\mathrm{mm}$. Hay cuentas tubulares de 9 a $51 \mathrm{~mm}$ de longitud. Se registraron 13 preformas, es decir, cuentas inacabadas de formas circulares planas o tubulares sin orificio $(\mathrm{N}=7)$ o con perforación incompleta en uno o ambos lados ( $\mathrm{N}=6)$ y piezas fragmentadas (fig. 5).

Difiere del resto una cuenta cuadrangular (PVRc5) que ha sido formatizada y tiene una perforación, pero 


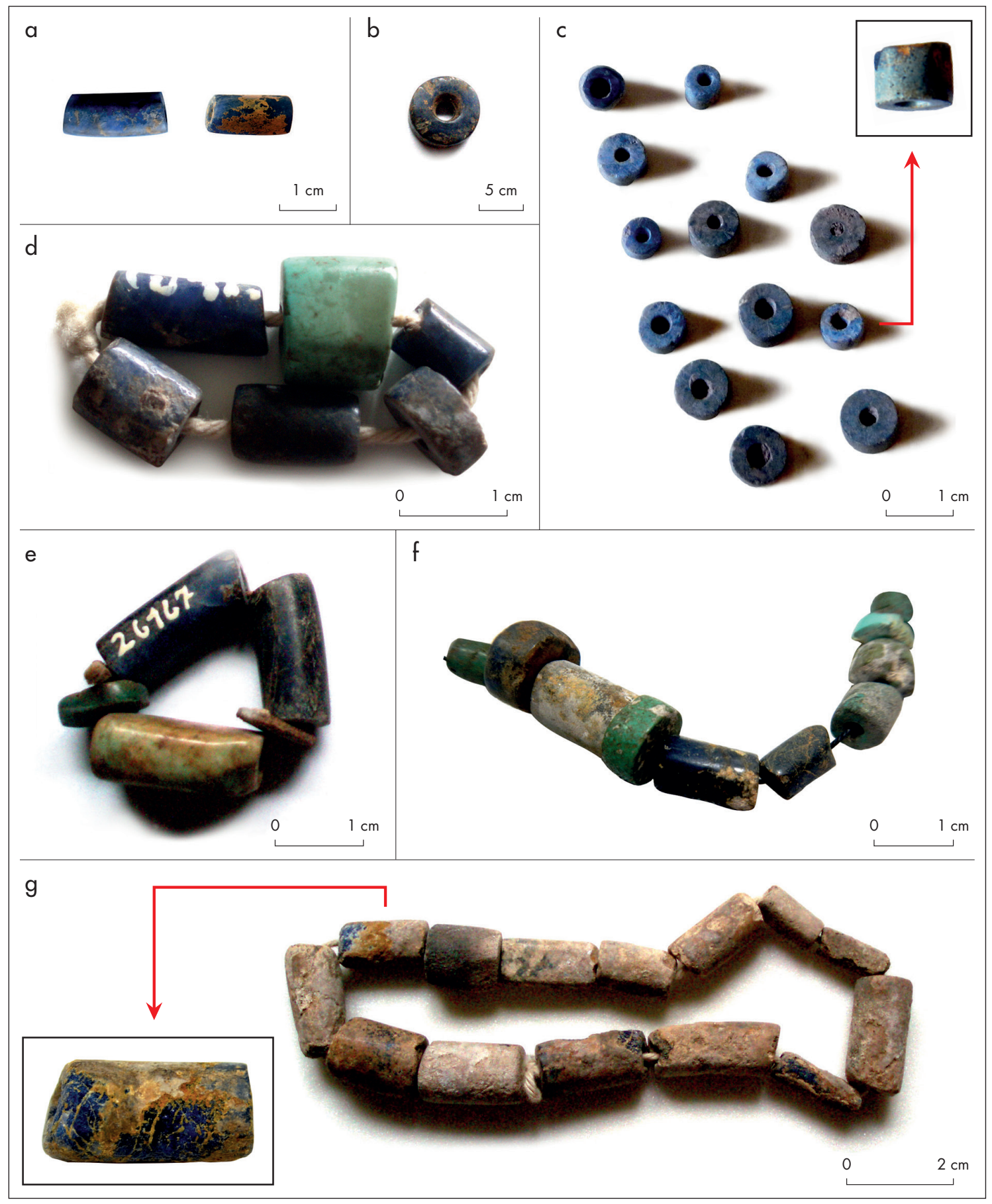

Figura 3. Cuentas confeccionadas con sodalita. El armado de los collares y pulseras es actual: a) PVRC27, recolección superficie; b) PVRC29, hallazgo en capa (E7 C1c2); c) PVRC28, colección privada; d) Titiconte, MLP-Ar-CMM-7341-25350 a 25355, junto con otras cuentas; e) PVRC, MLP-Ar-CMM-7936-26167, junto con otras cuentas; f) Titiconte, XxVE-ME-48211 (-37944-), junto con otras cuentas; g) PVRC4, MLP-Ar-CMM-7293-24934 a 25058, con taranakita. Figure 3. Beads made of sodalite. The assembly of the necklaces and bracelets is contemporary: a) PVRC27, surface collection; b) PVRC29, find in layer (E7 C1c2); c) PVRC28, private collection; d) Titiconte, MLP-ArCMM-7341-25350 to 25355, together with other beads; e) PVRC, MLP-Ar-CMM-7936-26167, together with other beads; $f$ ) Titiconte, XXVEME-48211 (-37944-), together with other beads; g) PVRC4, MLP-Ar-CMM-7293-24934 a 25058, with taranakite. 


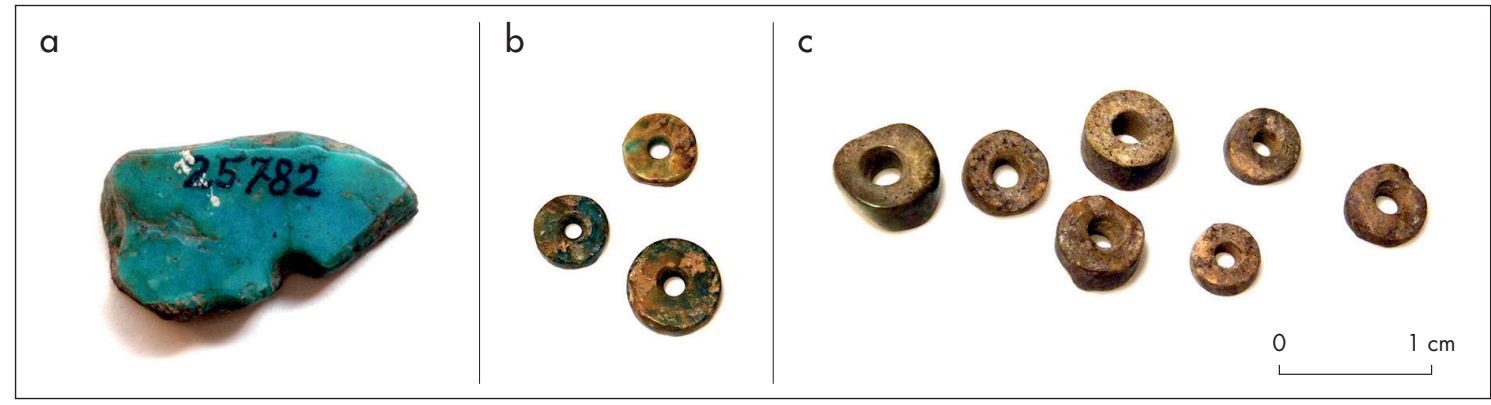

Figura 4. Cuentas confeccionadas con ópalo: a) Zapallar, Z100, MLP-Ar-CMM-7554-25782; b) PVRC11, MLP-Ar-CMM-7294-25059 a 25181; c) PVRC6, MLP-Ar-CMM-7932-26162. Figure 4. Beads made of opal: a) Zapallar, Z100, MLP-Ar-CMM-7554-25782; b) PVRC11, MLP-ArCMM-7294-25059 to 25181; c) PVRC6, MLP-Ar-CMM-7932-26162.

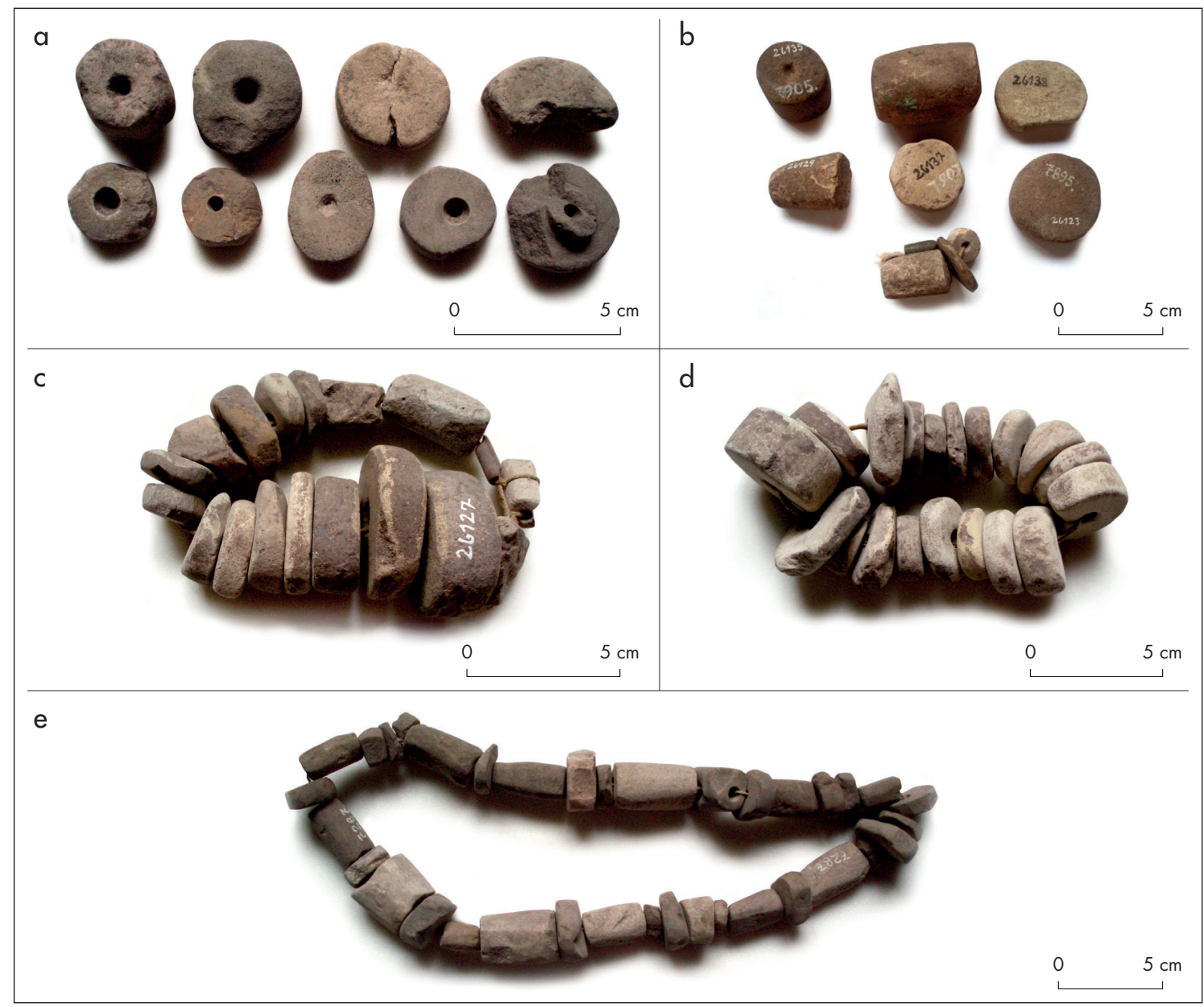

Figura 5. Preformas y cuentas líticas de PVRC. El armado de la pulsera es actual: a) MLP-Ar-CMM-7290-24860 a 24871; b) MLP-ArCMM-7905-26135, MLP-Ar-CMM-7908-26138, MLP-Ar-CMM-7895-26123, MLP-Ar-CMM-7907-26137, MLP-Ar-CMM-7899-26129, MLP -Ar-CMM-7909-26139, MLP-Ar-CMM-7299-25192 a 25195; c) MLP-Ar-CMM-7903-26133 (19 cuentas); d) MLP-Ar-CMM-7898-26127 (18 cuentas); e) MLP-Ar-CMM-7287-24684 a 24719. Figure 5. Preforms and lithic beads from PVRC. The assembly of the bracelet is contemporary: a) MLP-Ar-CMM-7290-24860 to 24871; b) MLP-Ar-CMM-7905-26135, MLP-Ar-CMM-7908-26138, MLP-Ar-CMM-7895-26123, MLP-ArCMM-7907-26137, MLP-Ar-CMM-7899-26129, MLP-Ar-CMM-7909-26139, MLP-Ar-CMM-7299-25192 to 25195; c) MLP-Ar-CMM-7903-26133 (19 beads); d) MLP-Ar-CMM-7898-26127 (18 beads); e) MLP-Ar-CMM-7287-24684 to 24719. 


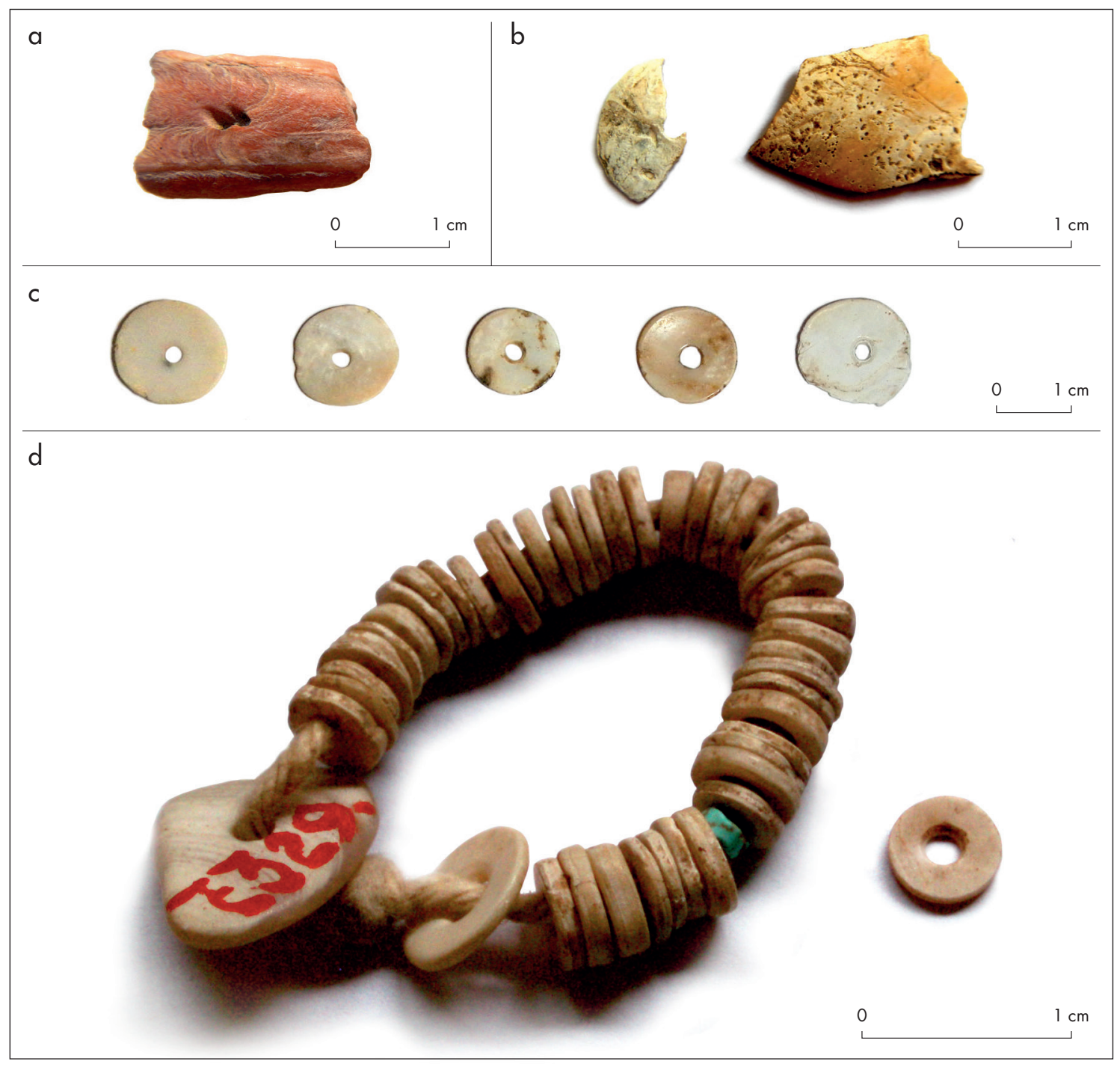

Figura 6. Cuentas malacológicas. El armado del collar es actual: a) cuenta de Spondylus sp. Cerro Morado, XxvE-ME-48228; b) fragmentos de cuenta y de Megalobulimus sp. Alero Mal Paso; c) cuentas de Megalobulimus sp. Alero Mal Paso; d) PVRC, MLP-Ar-CMM-7329-25231 a 25277. Figure 6. Malacological beads. The necklace assembly is contemporary: a) Spondylus sp. bead Cerro Morado, XXVE-ME-48228; $\boldsymbol{b}$ ) bead and Megalobulimus sp. fagments Alero Mal Paso; c) Megalobulimus sp. beads Alero Mal Paso; d) PVRC, MLP-Ar-CMM-7329-25231 to 25277.

está parcialmente fragmentada. Fue confeccionada en una roca atravesada por una venilla verde que se analizó mediante EDX, evidenciando altos porcentajes de $\mathrm{Cu}$, Ca y S (tabla 3).

\section{Cuentas malacológicas}

Se registraron 57 cuentas malacológicas. Son mayormente circulares planas con una perforación central, con diámetros de 5 a $14 \mathrm{~mm}$ y espesores de 0,5 a $1 \mathrm{~mm}$. Una excepción es la cuenta de Cerro Morado, rectangular (10 por $16 \mathrm{~mm}$ y $4 \mathrm{~mm}$ de espesor) de color rosa fuerte y manufacturada sobre Spondylus sp. (mullu).

Las cuentas de Alero Mal Paso fueron confeccionadas con Megalobulimus sp. (antes Strophocheilus sp.). En uno de sus lados tienen el brillo propio del gasterópodo. También se halló un fragmento sin formatizar que presenta una leve curvatura que se condice con ciertos sectores de la conchilla de este molusco (fig. 6). 


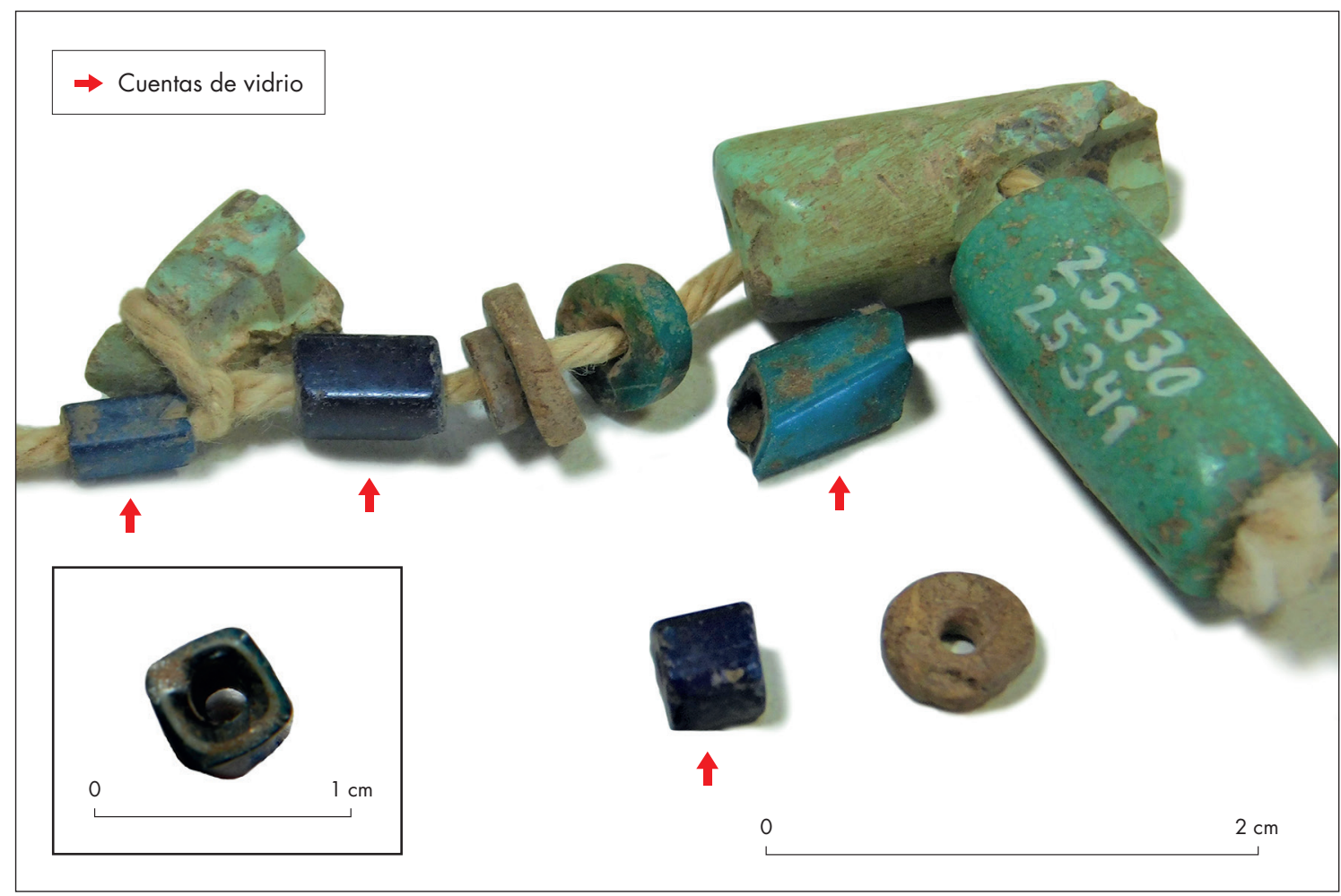

Figura 7. Cuentas de vidrio Nueva Cádiz, CA, MLP-Ar-смм-7332-25330 a 25341. Figure 7. Nueva Cádiz glass beads CA, MLP-ArCMM-7332-25330 to 25341.

\section{Cuentas de vidrio}

En Cuesta Azul se registraron cuatro cuentas de vidrio europeo conocidas como "Nueva Cádiz" que ingresaron tempranamente en el territorio peruano y que en el año 1560 fueron reemplazadas por estilos menos complejos y costosos (Sherr Dubin 1987: 258). Una es de color turquesa y tres de color azul oscuro. Todas son tubulares de sección cuadrangular (3-4 mm) y tienen entre 4 y $7 \mathrm{~mm}$ de largo. Han sido cortadas de piezas de mayor longitud (fig. 7).

\section{MATERIAS PRIMAS Y POSIBLES FUENTES DE PROVENIENCIA}

Los resultados obtenidos permiten realizar algunas consideraciones sobre las materias primas empleadas en la confección de cuentas y sus posibles fuentes de aprovisionamiento. En relación con las cuentas malacológicas, el hallazgo de piezas manufacturadas en
Megalobulimus sp. en un sitio a 4300 msnm resulta interesante. Este gasterópodo tiene una distribución muy amplia en el territorio argentino, desde las Yungas, los valles y el Chaco hasta áreas de pronunciada sequía. También podría ocupar pisos ecológicos más elevados, como los valles de la región en la cota de 3000 msnm (Sergio Miquel, comunicación personal 2018), pero es poco probable que esta especie ocupe espacios a más de $4000 \mathrm{msnm}$. Contrariamente, la cuenta de Spondylus sp. de Cerro Morado indica el traslado desde la costa del Pacífico, comparable con ofrendas registradas en otros santuarios de altura incas (Reinhard \& Ceruti 2010).

Las cuentas líticas en toba, pizarra, lutita y probable caliza pudieron ser manufacturadas localmente al tener los pobladores del área fácil acceso a estas materias primas. Las preformas de cuentas de toba registradas apoyan esta propuesta. Es diferente el caso de las cuentas minerales. A continuación, nos referiremos a posibles fuentes de aprovisionamiento de cada mineral identificado -turquesa, sodalita y ópalo- a partir de bibliografía geológica. 


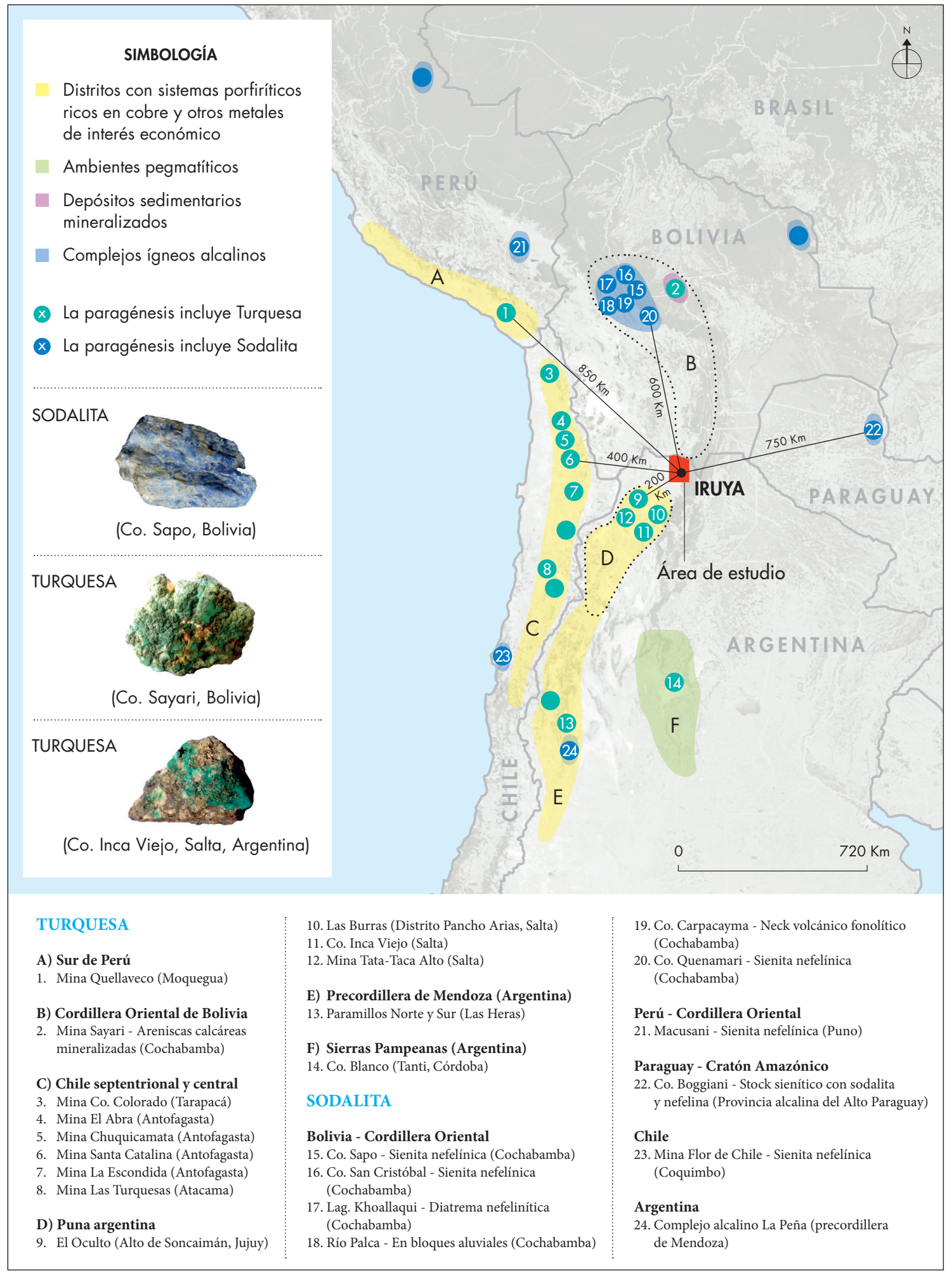

Figura 8. Ubicación de las principales fuentes de turquesa y sodalita en la macro-región centro meridional de América del Sur. Figure 8. Location of the main sources of turquoise and sodalite in the south-central macro-region of South America 
Turquesa: es un hidrofosfato de cobre y aluminio de color azul-verde (dureza: 5 a 6 ). Esta piedra poco común ha sido valorada desde la antigüedad como gema debido a las llamativas tonalidades que suele presentar, desde azul oscuro, azul claro y azul verdoso a verde manzana, amarillo verdoso y gris verdoso, además de una amplia variedad de tonalidades intermedias. $\mathrm{Su}$ fórmula química $\left[\mathrm{CuAl}_{6}\left(\mathrm{PO}_{4}\right)_{4}(\mathrm{OH})_{8} \bullet 4 \mathrm{H}_{2} \mathrm{O}\right]$ puede variar al formar series minerales con extremos ricos en $\mathrm{Al}$ (III) o Fe (III). Su estructura molecular permite la inclusión de calcio $(\mathrm{Ca})$, magnesio $(\mathrm{Mg})$, manganeso $(\mathrm{Mn})$, silicio $(\mathrm{Si})$, cromo $(\mathrm{Cr})$ y zinc $(\mathrm{Zn})$. La turquesa es un mineral secundario formado habitualmente en la zona de oxidación de pórfiros cupríferos afectados por hidrotermalismo; cristaliza rellenando intersticios, oquedades y venillas. También se origina por la acción de las aguas meteóricas actuantes sobre rocas ígneas aluminosas y sedimentarias ricas en fosfatos (Hudson Institute of Mineralogy 1993-2019, Foord \& Taggart Jr. 1998).

Los trabajos mineros refieren la presencia de numerosos pórfiros cupríferos localizados en la margen occidental de América del Sur (Bertrand et al. 2014). Nuestro interés se focalizó en los cuerpos porfíricos portadores de sulfuros de cobre con formación de oxidados (entre ellos, turquesa) ubicados en las montañas de Chile, Perú, Bolivia y Argentina (fig. 8).

En el norte y centro de Chile se ubica la mayoría de los depósitos cupríferos con turquesa, algunos con evidencias de explotación prehispánica como Chuquicamata, minas Las Turquesas y El Abra (Salazar 2008, Salazar et al. 2013, González Godoy et al. 2017).

En Perú se registran numerosos cuerpos de cobre porfírico (Bertrand et al. 2014, Martínez-Valladares et al. 2017); sin embargo, es escasa la información disponible sobre la presencia de depósitos que incluyan turquesa, siendo la mina Quellaveco (Moquegua) la única fuente mencionada en la bibliografía especializada (Singer et al. 2008). Reindel y colaboradores (2013: 307) señalan que en 1982 el geólogo Hans Ruppert postuló que en el sur de Perú había fuentes de turquesa no registradas aún. Stöllner y colaboradores (2013: 125) mencionan la existencia de varios depósitos de turquesa en Palpa, sugiriendo la posibilidad de que, en esa localidad o en la región de Nazca en general, se encontrase una de las fuentes de turquesa peruana. No obstante, la turquesa cultural fue históricamente en Perú la crisocola -silicato de cobre- (Petersen 1970).
En Bolivia, no obstante los importantes yacimientos mantiformes con mineralización de cobre (minas Coro Coro y Chacarilla, Departamento La Paz), se cuenta con escasa información sobre la existencia de fuentes de turquesa (Arce Burgoa 2007, Serrano Bravo 2004). El único dato geológico/minero actual en el que se menciona turquesa se refiere al depósito sedimentario de areniscas calcáreas mineralizadas del cerro Sayari (mina Sayari, Cochabamba) (Hudson Institute of Mineralogy 1993-2019).

A diferencia de lo postulado previamente desde la arqueología, en la región andina argentina, varios cuerpos porfíricos polimetálicos también contienen turquesa como mineral secundario. Entre ellos, los distritos mineros puneños Alto de Soncaimán/mina El Oculto (Jujuy), Taca-Taca (Salta), Pancho Arias/mina Las Burras (Salta) y Cerro Inca Viejo (Salta). También se menciona turquesa en el yacimiento El Pachón (cordillera Principal, Calingasta, San Juan) y en las minas de Paramillos Norte y Sur (precordillera, Las Heras, Mendoza) (Chabert 1999, Godeas et al. 1999, Rubinstein et al. 2000, Singer et al. 2008, Carrasquero 2015). Los recientes hallazgos de López y colaboradores (2018) en Cueva Inca Viejo (Salta) significan un valioso aporte que pone fin a una etapa en la que se consideraba que toda turquesa hallada en sitios arqueológicos del territorio argentino era alóctona y provenía del norte de Chile. En este yacimiento de la puna salteña hay evidencias de explotación de turquesa que se remontan principalmente a momentos incaicos.

Sodalita (feldespatoide): es un aluminosilicato rico en sodio. Químicamente, es similar a los feldespatos alcalinos, aunque deficiente en sílice. Su fórmula química es $\mathrm{Na}_{4} \mathrm{Al}_{3} \mathrm{Si}_{3} \mathrm{O}_{12} \mathrm{Cl}$; no obstante, presenta variaciones por ser parte de la serie sodalita-noseana-haüyna-lazurita (sodalita con $\mathrm{Na}$ y $\mathrm{Cl}$, noseana con $\mathrm{Na}$ y $\mathrm{S}$, y haüyna y lazurita con Na-Ca y S). La sodalita se presenta en distintos colores -incoloro, blanco, gris, verde, azul celeste, rosa y rojo-, pero las variedades más llamativas son azules a celeste-liláceas. Este mineral (dureza: 5,5 a 6 ) ocurre en rocas ígneas que cristalizaron a partir de magmas alcalinos subsaturados en sílice. La sodalita se encuentra como relleno de venas y fisuras en sienitas nefelínicas, traquitas y fonolitas, aunque también existen registros en calizas (Anthony et al. 1995, King 2005-2019).

En la cordillera Oriental de Bolivia se ubica el Complejo Alcalino de Cerro Sapo con interesantes 
asociaciones minerales; entre ellas, el sistema de diques sienito-nefelícos portadores de sodalita-ankeritabaritina (Schultz et al. 2004). Se ha sostenido que las gemas de sodalita registradas en Tiahuanaco y en sitios preincaicos fueron obtenidas en la región de Ayopaya (Browman 1998: 308). Las sienitas nefelínicas de los cerros San Cristóbal y Quenamari, pertenecientes al mismo complejo alcalino, también contienen sodalita, al igual que la fonolita del cerro Carpacayma (Ahlfeld 1946: 158). Asimismo, se menciona sodalita en las sienitas nefelínicas de la Provincia Alcalina Velazco (Santa Cruz, cerca del límite con Brasil) (Comin-Chiaramonti et al. 2005) (fig. 8).

En Perú se ha detectado sodalita en la sienita nefelínica de Macusani (Puno) y en las fonolitas y traquitas alcalinas de Pucallpa (Loreto) (Stewart 1971, Laubacher 1978).

En el NOA se han registrado numerosos diques y cuerpos con afinidades alcalinas, desde granitos, sienitas y fonolitas nefelínicas hasta diques ultrabásicos que incluyen minerales alcalinos, aunque en ningún caso se ha mencionado sodalita. Los cuerpos alcalinos más cercanos a los sitios arqueológicos estudiados serían dos diques de tinguaíta ubicados $25 \mathrm{~km}$ al nor-noroeste de Iruya (y a $18 \mathrm{~km}$ al noroeste de Rodeo Colorado, Salta). La roca sería del mismo tipo magmático que la sienita nefelínica de Cerro Sapo (Quartino 1960: 155); no obstante, la paragénesis publicada no incluye sodalita. Ejemplos similares son las alnöítas de Los Alisos (Iruya), $50 \mathrm{~km}$ al sudeste de Iruya (Villar et al. 1997), la tinguaíta de Cobres (Puna salteña) descrita por Hausen (1930), las rocas alcalinas -basanitas, bostonitas, limburgitas y traquiandesitas- cercanas al cerro Hornillos (Sierra de Santa Victoria, Salta) mencionadas por Beder en 1928, además de otras localidades con manifestaciones básicas alcalinas de Tucumán y Catamarca (Quartino 1960: 152-153, Rubiolo 1997). En cambio, se menciona sodalita en el Complejo Alcalino La Peña en la precordillera mendocina -ca. $1200 \mathrm{~km}$ de Iruya (Pagano et al. 2016) - y en otras localidades del sur argentino, más alejadas aún (fig. 8).

Hemos mencionado que algunas cuentas de sodalita presentan una costra de color castaño claro. El estudio DRX realizado previamente indicó taranakita $\left[(\mathrm{K}, \mathrm{Na})_{3}(\mathrm{Al}, \mathrm{Fe})_{5}\left(\mathrm{PO}_{4}\right)_{2}\left(\mathrm{PO}_{3} \mathrm{OH}\right)_{6} \cdot 18 \mathrm{H}_{2} \mathrm{O}\right]$, un fosfato hidratado de aluminio, hierro (III) y álcalis que suele precipitar formando agregados nodulares pulverulentos, masivos o costras; su color varía entre amarillo, gris y blanco (dureza: 1-2). Se forma bajo condiciones ácidas y húmedas a partir de la interacción entre las soluciones fosfáticas, resultantes de la degradación del guano (de aves o murciélagos) o de restos óseos acumulados en cuevas, y el potasio y el aluminio derivados del sustrato (Mineral Data Publishing 2001-2005, Giurgiu et al. 2013). Aunque no contamos con información sobre el contexto de hallazgo de estas cuentas, es altamente probable que provengan de entornos funerarios. Posiblemente, fueron depositadas en entierros como ofrendas o adornos y entraron en contacto con los huesos, lo cual explicaría la formación de taranakita sobre algunas de las piezas de sodalita como resultado de procesos posdepositacionales $(\mathrm{N}=20)$ (fig. 3 ).

Ópalo: es un mineraloide silíceo; químicamente es sílice coloidal precipitada $\left[\mathrm{SiO}_{2} \bullet \mathrm{nH}_{2} \mathrm{O}\right]$. Su dureza varía entre 5,5 y 6,5 y, en su estado puro, es incoloro o blanco lechoso con opalescencia perlada, aunque también puede ser amarillo, marrón, verde, anaranjado y azul. El ópalo se forma a partir de soluciones ricas en sílice a temperaturas relativamente bajas y puede encontrarse rellenando fisuras y cavidades en una gran variedad de rocas. Es un componente común en infinidad de localidades en la macro-región, dificultando la localización de posibles fuentes de aprovisionamiento. Sin embargo, el ópalo de color turquesa (muestra Z100) analizado previamente (Ventura \& Scambato 2013) podría estar relacionado con cuerpos cupríferos. En las cercanías de los sitios estudiados, hay yacimientos de cobre que no hemos investigado aún, motivo por el cual no descartamos un origen local.

\section{CONSIDERACIONES FINALES}

Los resultados obtenidos hasta el momento permitieron caracterizar un conjunto de cuentas de las que, previamente, contábamos solo con una descripción somera e inexacta. A partir de este estudio observamos que las cuentas recuperadas en sitios arqueológicos de la región son líticas (mayoritariamente toba), de material malacológico y de vidrio, aunque la gran mayoría (casi el $72 \%)$ han sido confeccionadas con minerales: turquesa (66\%), sodalita (4,9\%) y, en muchísima menor proporción, ópalo y posiblemente crisocola. Llama la atención el predominio casi exclusivo de cuentas de turquesa, mineral que, como mencionamos anteriormente, ha 
sido altamente valorado en momentos prehispánicos y especialmente incas. Lamentablemente la información contextual es escasa y la cronología de las cuentas, en sitios multicomponentes, como PVRC, de donde proviene la mayor cantidad y variedad de estas piezas, resulta difícil de precisar. Es destacable, no obstante, que en las colecciones analizadas se encuentren materiales asignables a momentos incas (cerámica, ciertas piezas de plata como placas circulares y una pulsera decorada, y cubiletes de oro, entre otras) que, sumadas a la información etnohistórica, dan cuenta de grupos jerarquizados con acceso a bienes de gran valor simbólico en el Tawantinsuyu, entre los cuales también se podrían incluir las cuentas minerales analizadas. No es menor el hallazgo de cuentas de vidrio Nueva Cádiz, recuperadas en otros sitios del NOA con importantes ocupaciones incas previas, cuya población tuvo acceso a bienes sofisticados y costosos (Tarragó 1984, Raffino \& Palma 1993, Bordach 2006).

En el análisis del conjunto de cuentas, es importante hacer una distinción entre aquellas que pudieron haber sido producidas localmente y las que fueron confeccionadas con materias primas alóctonas y arribaron al sitio como productos terminados, a través del intercambio, o, si fuera el caso, actuando como dones o regalos para individuos o grupos elegidos por el Inca (Ventura \& Scambato 2013). En cuanto a las cuentas líticas, la geología nos indica que el aprovisionamiento pudo darse en la misma región o regiones vecinas, y su manufactura haber sido local, dada la presencia de piezas en distintas etapas de confección (sin orificios, con orificios sin terminar, fragmentadas y completas).

Las cuentas malacológicas (Megalobulimus sp.) del Alero Mal Paso también pudieron haber sido de manufactura local. La recolección de este gasterópodo pudo haberse dado en los valles de la región (3000 $\mathrm{msnm}$ ) donde se encuentran los asentamientos residenciales mencionados. Un fragmento de ese material sin formatizar junto a dichas cuentas lo sugiere, aunque es escasa la evidencia para confirmarlo.

Respecto de las cuentas minerales, las piezas se encuentran completas o fragmentadas, pero nunca como preformas. Fueron, además, manufacturadas en materias primas aparentemente no existentes en las cercanías de los sitios. Según el detalle de yacimientos de turquesa y sodalita que presentamos en este trabajo, los mismos se localizan entre 200 y $850 \mathrm{~km}$ de distancia en línea recta de estos asentamientos. Para el caso de la turquesa, aunque su origen se redujo tradicionalmente en la literatura arqueológica argentina a los reconocidos yacimientos del actual norte chileno, los recientes hallazgos en Cueva Inca Viejo (Puna de Salta) y el análisis de bibliografía geológica realizada en esta oportunidad abren un panorama mayor en relación con potenciales fuentes de proveniencia, necesario para cualquier abordaje sobre estas temáticas. Dicho yacimiento se encuentra a $315 \mathrm{~km}$ de PVRC y allí han sido identificadas labores de extracción de turquesa en momentos prehispánicos (López et al. 2018). En este yacimiento se destaca la importancia simbólico-ritual que le otorgaba el Tawantinsuyu a las minas de turquesa, cuyo registro arqueológico refiere también a tráfico macro-regional y a prácticas rituales, entre cuyos materiales se cuentan algunos provenientes de los valles mesotermales y de las Yungas (Coloca 2020).

En los otros pórfiros cupríferos portadores de turquesa de la puna salteña y jujeña, aún no se han registrado evidencias de explotación antigua, aunque son áreas con una larga historia de ocupación humana, relativamente cerca de las cuales se han identificado cuentas de este mineral. Nos referimos a los contextos funerarios de Queta y Pucará de Rinconada en la puna jujeña, y de Gólgota y Tastil en la quebrada del Toro, Salta (Boman 1908).

La sodalita, en cambio, no ha sido detectada aún en las Sierras Orientales argentinas (prolongación meridional de la cordillera Oriental boliviana), ni en las provincias geológicas vecinas con evidencias de magmatismo alcalino, aunque cuentas de este mineral se han encontrado en cementerios de la selva pedemontana salteña, datados alrededor del 1000 AP (Ventura 1991) y de Tilcara, asignables a la mitad del primer milenio de la era (López Campeny 2012). También en el área de Calahoyo, Cieneguillas y Santa Catalina, Jujuy, según Fernández y Menzel (1980), y en Tolomosa, al sur de Tarija, en Bolivia (Von Rosen (1957 [1919]). ${ }^{6}$ Considerando la bibliografía, los especímenes mejor conocidos de América del Sur provendrían de la región de Ayopaya, principalmente de Cerro Sapo en Bolivia, a $650 \mathrm{~km}$ de PVRC, si bien hay registros de otras fuentes de sodalita en Perú, Paraguay y Chile, incluso en Mendoza, Argentina. Estos datos nos permiten pensar en el traslado a larga distancia de minerales y productos terminados a los que accedieron los habitantes de los valles orientales, aunque los mecanismos aún deben ser estudiados. En el caso de que las cuentas hubiesen arribado a los asentamientos durante momentos incas, las posibilidades se amplían 
aún más, dada la enorme capacidad de movilización de bienes y materias primas del Tawantinsuyu. Un ejemplo de ello es el hallazgo en Cerro Morado de la cuenta de Spondylus sp. proveniente del Pacífico e identificada en este trabajo.

Para finalizar, es importante señalar que, si bien es necesario ahondar en los estudios para resolver los interrogantes abiertos en este artículo, este trabajo significa un primer avance en este sentido, caracterizando de manera no destructiva valiosas piezas de colecciones de museos y de campo que hasta esta investigación no habían sido analizadas geoquímicamente, y reuniendo datos sobre fuentes minerales no mencionadas en la literatura arqueológica disponible.

Agradecimientos Agradecemos a Sergio Miquel (CONicet, Museo de Ciencias Naturales B. Rivadavia, Buenos Aires), Mateo Quispe y a las comunidades de Abra del Sauce y Rodeo Colorado, Salta; a Laura Miotti y Mariano Bonomo, División Arqueología del Museo de La Plata, a Virginia Villamayor, y a los encargados y personal de los depósitos, Ana Igareta, Jorgelina Collazo y Gabriel Alarcón; a Gabriela Ammirati, Alejandra Reynoso y Mónica Berón del Museo Etnográfico; a Gabriel López, Marisa López Campeny, Marcela Sepúlveda, James Brennan, Luis Borrero, Carlos Angiorama. A los evaluadores anónimos y a CONICET, CNEA y CIGA-SEGEMAR.

\section{NOTAS}

${ }^{1}$ Las limitaciones del análisis macroscópico basado en el color y la necesidad de determinaciones geoquímicas han sido también señaladas en el caso de cuentas recuperadas en el norte de Chile (Westfall et al. 2010, Carrión 2015).

${ }^{2}$ La CMM-MLP correspondiente a los valles orientales del norte de Salta se compone de más de 1000 fichas de piezas individuales y de lotes con las cuales Granda (2010) confeccionó un inventario digital.

${ }^{3}$ De acuerdo con las fichas del Museo, dentro de los diez lotes no hallados, habría cuatro cuentas de PVRC, ocho cuentas sin especificar sitios (Departamentos Iruya y Santa Victoria) y lotes con un número desconocido de cuentas de PVRC, Huaira Huasi, Cuesta Azul y Zapallar.

${ }^{4}$ El número de muestras analizadas de esta colección estuvo condicionado por los plazos establecidos por el Museo para la devolución de las piezas.

${ }^{5}$ Dos de estas cuentas fueron analizadas petrográficamente por Virginia Villamayor (MLP) en 2012.

${ }^{6}$ Incluimos solo aquellos que han sido identificados mediante estudios geoquímicos.

\section{REFERENCIAS}

Ahlfeld, F. 1946. Geología de Bolivia. Revista del Museo de La Plata, Nueva Serie, Sección Geología 3 (19): 5-370.

Anthony, J., Bideaux, R., Blath, K. \& Nichols, M. 1995. Handbook of mineralogy. Vol. II, Silica and Silicates. Tucson Arizona: Mineral Data Publishing.

Arce BurgoA, O. 2007. Guía a los yacimientos metalíferos de Bolivia. La Paz: SPC Impresores.

Berenguer, J. 2004. Caravanas, interacción y cambio en el Desierto de Atacama. Santiago: Sirawi.

Berthelot, J. 1978. L'exploitation des métaux précieux au temps des incas. Annales. Économies, Sociétés, Civilisations 33 (5-6): 948-966.

Bertrand, G., Guillou-Frottier, L. \& Loiselet, C. 2014. Distribution of porphyry copper deposits along the western Tethyan and Andean subduction zones: insights from a paleotectonic approach. Ore Geology Reviews 60: 174-190.

Boman, E. 1908. Antiquités de la region andine de la Republique Argentine et du désert D’Atacama. Paris: Masson.

BoRDACH, M. 2006. Interacciones étnicas e indicadores de desigualdad social en el cementerio de La Falda (SJTil43) de Tilcara, Jujuy. Estudios Atacameños 31: 115-128.

Brooks, W., Farfan, A., Vetter, L. \& Diestra, D. 2013. Proyectiles de plomo en el antiguo Perú. Bulletin de I'Institut Français d'Études Andines 42 (1): 13-23.

BROWMAN, D. 1998. Lithic provenience analysis and emerging material complexity at Formative Period Chiripa, Bolivia. Andean Past 5: 301-324.

Brown, A. \& GraU, R. 1993. La naturaleza y el hombre en las Selvas de Montaña. Salta, proyecto GTz. Salta: Sociedad Alemana de Cooperación Técnica-Proyecto Desarrollo Agroforestal en Comunidades rurales del Noroeste Argentino.

Cantarutti, G. 2013. Mining under Inca rule in North-Central Chile: the Los Infieles mining complex. In Mining and quarrying in the ancient Andes. Sociopolitical, economic and simbolic dimensions, N. Tripcevich \& K. Vaughn, eds., pp. 185-211. New York: Springer.

CARrasquero, S. 2015. Caracterización petrológica y geoquímica del volcanismo terciario en la región de Uspallata y su relación con las mineralizaciones hidrotermales del distrito minero Paramillos de Uspallata, Mendoza, Argentina. Thesis for the degree of Doctor in Natural Sciences, Facultad de Ciencias Naturales y Museo, Universidad Nacional de La Plata, La Plata.

CARrión, H. 2015. Cuentas de collar, producción e identidad durante el Período Medio en San Pedro de Atacama. In El Horizonte Medio: nuevos aportes para el sur de Perú, norte de Chile y Bolivia, A. Korpisaari \& J. Chacama, eds., pp. 265-280. Arica: Instituto Francés de Estudios AndinosUniversidad de Tarapacá.

CasAnova, E. 1930. Excursión arqueológica al Cerro Morado. Notas del Museo Etnográfico 3: 5-40. 
Chabert, M. 1999. El pórfiro cuprífero Inca Viejo, Salta. In Recursos minerales de la República Argentina, Anales 35, E. Zappetini, ed., pp. 1425-1436. Buenos Aires: SEgEmar.

Coloca, F. 2020. El Tawantinsuyu y la minería lapidaria en el Noroeste Argentino. Evidencias incaicas relacionadas con la explotación de turquesa en la Cuenca de Ratones, Puna de Salta. Revista del Museo de Antropología 13 (1): 51-56.

Comin-Chiaramonti, P., Gomes, C. B., Velázquez, V. F., Censi, P., Antonini, P., Comin-Chiaramonti, F. \& Punturo, R. 2005. Alkaline complexes from Southeastern Bolivia. In Mesozoic to Cenozoic Alkaline Magmatism in the Brazilian Platform, P. Comin-Chiaramonti \& C. B. Gomes, eds., pp. 159-213. São Paulo: EDUsP PublisherUniversidad de San Pablo.

Debenedetti, S. \& Casanova, E. 1933-1935. Titiconte. Publicaciones del Museo Antropológico y Etnográfico de la Facultad de Filosofía y Letras, Universidad de Buenos Aires, serie A, III: 1-35.

Fernández, J. \& Menzel, M. 1980. Sodalita en el Noroeste Argentino. Implicancias cronológicas y culturales. Anales de la Sociedad Científica Argentina 42: 37-43.

Foord, E. \& TAgGart JR., J. 1998. A reexamination of the turquoise group: the mineral aheylite, planerite (redefined), turquoise and coeruleolactite. Mineralogical Magazine 62 (1): 93-111.

GARrido, F. 2017. Comunidades mineras prehispánicas de pequeña escala y sus ventajas económicas y logísticas derivadas de su conectividad con el Qhapaq Nan, desierto de Atacama, norte de Chile. Boletín de Antropología 30: 48-74.

GiurgiU, A., OnaC, B., TĂmaş, T. \& Fornós, J. 2013. Evolution of guano under different environmental conditions: a mineralogical approach. In Proceedings of the 16th International Congress of Speleology, vol. 3, M. Filippi \& P. Bosák, eds., pp. 483-485. Brno: Czech Speleological Society.

Godeas, M., Cardó, R., Carrizo, R., Zuloeta, C., González JR., R., Korzeniewski, L., López, H., Mallimacci, H., Martínez, L., Ramallo, E., Valladares, H. \& Zubia, M. 1999. Inventario de yacimientos y manifestaciones de minerales metalíferos e industriales de la República Argentina. In Recursos minerales de la República Argentina, Anales 35, E. Zappettini, ed., pp. 1979-2172. Buenos Aires: SEgEMAR.

González, C. \& Westfall, C. 2008. Atacameños en El Salvador: nuevas apreciaciones sobre el fardo funerario del cementerio Las Turquesas. Estudios Atacameños 35: 49-73.

González Godoy, C., Westfall, C. \& Castells Schencke, C. 2017. Mina Las Turquesas: lapidaria, secuencia alfarera prehispánica e interrelaciones culturales en un espacio intermodal del desierto de Atacama, Chile. Estudios Atacameños 56: 225-251.

Granda, P. 2010. Paisaje y arquitectura en la arqueología de los valles orientales del norte de Salta (Argentina). Thesis for the Bachelor's degree in Anthropological Sciences, Facultad de Filosofía y Letras, Universidad de Buenos Aires, Buenos Aires.

Horta Tricallotis, H. 2008. Insignias para la frente de los nobles incas: una aproximación etnohistórica-arqueológica al principio de la dualidad. In Lenguajes visuales de los incas, P. González \& T. Bray, eds., vol. 1848, pp. 71-89. Oxford: BAR International Series.

Hudson Institute of Mineralogy. 1993-2019. Turquoise. $<$ https://www.mindat.org/min/turquoise.html > [consultado: 02-10-2018].

KING, H. 2005-2019. Sodalite. The blue gem material, ornamental and architectural stone. <https://geology.com/minerals/ sodalite.shtml> [consultado: 01-10-2018].

LAUBACHER, G. 1978. Estudio geológico de la región norte del lago Titicaca. Boletín del Instituto de Geología y Minería 5 (D): 1-122.

López, G., Coloca, F., Rosenbusch, M. \& Solá, P. 2018. Mining, macro-regional interaction and ritual practices in the South-Central Andes: the first evidence for turquoise exploitation from the late prehispanic and Inca Periods in north-western Argentina (Cueva Inca Viejo, puna of Salta). Journal of Archaeological Science Reports 17: 81-92.

LóPEZ CAMPENy, S. 2012 Ms. Rescate Carrazana-Paredes (Barrio Malka, Tilcara, Jujuy). Análisis del conjunto de cuentas recuperadas. Informe para Patrimonio de la Provincia de Jujuy, Argentina.

Márquez Miranda, F. 1939. Cuatro viajes de estudio al más remoto noroeste argentino. Revista del Museo de La Plata, Sección Antropología 1 (6): 93-243.

Martínez-Valladares, W., Marchena-Campos, A., OteroAguilar, J., Cervantes-Gárate, J. \& León-Lecaros, W. 2017. Geología y controles tectonomagmáticos de los sistemas porfiríticos en el Arco Magmático Occidental del Sur de Perú. Lima: Instituto Geológico Minero y MetalúrgicoDirección de Recursos Minerales y Energéticos-Programa de Metalogenia.

Mineral Data Publishing. 2001-2005. Taranakite. <http:// www.handbookofmineralogy.org/pdfs/taranakite $>$ [consultado: 05-10-2018].

Nielsen, A., Angiorama, C. \& Ávila, F. 2017. Ritual as interaction with non-humans: prehispanic mountain pass shrines in the Southern Andes. In Rituals of the past: prehispanic and colonial case studies in andean archaeology, S. Rosenfeld \& S. Bautista, eds., pp. 241-266. Boulder: University Press of Colorado.

Pagano, D., Galliski, M., Márquez-Zavalía, M. \& CoLомво, F. 2016. Petrology and mineralogy of the La Peña igneous complex, Mendoza, Argentina: an alkaline occurrence in the Miocene magmatism of the Southern Central Andes. Journal of South American Earth Sciences 67: 158-179.

Petersen, G. 1970. Minería y metalurgia en el antiguo Perú. Vol. 12. Lima: Instituto de Investigaciones Antropológicas. 
QuARTino, B. 1960. Acerca de una tinguaíta de Iruya (Provincia de Salta) y de las rocas alcalinas del noroeste argentino. Revista de la Asociación Geológica Argentina xv (3-4): 145-157.

Raffino, R., Alvis, R., Olivera, D. \& Palma, J. 1986. La instalación inka en la sección andina meridional de Bolivia y extremo boreal de Argentina. Comechingonia 4: 63-131.

Raffino, R. \& PAlma, J. 1993. Los artefactos. In Inka. Arqueología, historia y urbanismo del altiplano andino, R. Raffino, ed., pp. 93-129. Buenos Aires: Ediciones Corregidor.

RAPP, G. 2009. Archaeomineralogy. Heidelberg: Natural Science in Archaeology Series-Springer Verlag.

Reindel, M., Stöllner, T. \& GräFingholt, B. 2013. Mining archaeology in the Nasca and Palpa Region, South Coast of Peru. In Mining and quarrying in the ancient Andes. Sociopolitical, economic and symbolic dimentions, N. Tripcevich \& K. Vaughn, eds., pp. 299-322. New York: Interdisciplinary Contributions to Archaeology.

Reinhard, J. \& Ceruti, C. 2010. Inca rituals and sacred mountains. A study of the world's highest archaeological sites. Los Angeles: Cotsen Institute of Archaeology Press, University of California.

Rubinstein, N., Segal, S. \& Zappettini, E. 2000. El pórfiro cuprífero Taca-Taca Alto, Provincia de Salta: nuevos aportes al conocimiento del yacimiento. Revista de la Asociación Geológica Argentina 55 (1-2): 125-128.

Rubiolo, D. 1997. Alkaline rocks in Central Andes from NW-Argentina and Bolivia: Tectonic implications. Actas del viII Congreso Geológico de Chile, vol. 3, pp. 1719-1723. Antofagasta: Universidad Católica del Norte.

Rubiolo, D., Seggiaro, R., Gallardo, E., Disalvo, A., Sánchez, M., Turel, A., Ramallo, E., Sandruss, A. \& GodeAs, M. 2003. Hoja geológica 2366-II/2166-IV La Quiaca, Provincias de Jujuy y Salta. Boletín 246. Buenos Aires: Instituto de Geología y Recursos Minerales, SEgEMAR.

SAlazAR, D. 2008. La organización de la producción minera en San José del Abra durante el Período Tardío atacameño. Estudios Atacameños 36: 43-72.

Salazar, D., Berenguer, J. \& Vega, G. 2013. Paisajes minerometalúrgicos inkaicos en Atacama y el altiplano sur de Tarapacá (norte de Chile). Chungara 45 (1): 83-103.

Schultz, F., Lehmann, B., Tawackoli, S., Rössling, R., Belyatsky, B. \& Dulski, P. 2004. Carbonatite diversity in the Central Andes: the Ayopaya alkaline province, Bolivia. Contributions to Mineralogy and Petrology 148 (4): 391-408.

Serrano Bravo, C. 2004. Historia de la minería andina boliviana (siglos XVI-XX). <https://docplayer.es/12207395Historia-de-la-mineria-andina-boliviana-siglos-xvi-xx. html> [consultado: 06-10-2018].

SHerr Dubin, L. 1987. The history of beads from 30.000 BC to the present. New York: Harry Abrams.

Singer, D., Berger, V. \& Moring, B. 2008. Porphyry copper deposits of the world: database, map, and grade and tonnage models. US Geological Survey Open-File Report 2008-
1155. <http://pubs.usgs.gov/of/2008/1155> [consultado: 02-10-2018].

Soто, C. 2019. "Objetos perforados" asociaciones simbólicas y redes de circulación: reflexiones sobre las formas de intercambio en el Período Formativo (1500 AC-500 BC) del desierto de Atacama, norte de Chile. Chungara 51 (4): 573-593.

Stewart, J. 1971. Neogene peralkaline igneous activity in Eastern Peru. Geological Society of America Bulletin 82 (8): 2307-2312.

Stöllner, T., Reindel, M., Gassman, G., Gräfingholt, B. \& Isla Cuadrado, J. 2013. Precolumbian raw-material exploitation in Sourthern Peru. Structures and perspectives. Chungara 45 (1): 105-129.

TARragó, M. 1984. El contacto hispano-indígena. La provincia de Chicoana. Runa 14: 143-185.

VenturA, B. 1991. Síntesis de las investigaciones arqueológicas en el sector norte de las selvas occidentales. Arqueología 1: 51-73.

Ventura, B. \& Scambato, A. 2013. La metalurgia de los valles orientales del norte de Salta, Argentina. Boletín del Museo Chileno de Arte Precolombino 18 (1): 85-106.

Ventura, B. \& Oliveto, G. 2014. Resabios de otros tiempos. Dominio incaico en los valles orientales del norte de Salta, Argentina. Bulletin de l'Institut Français d'Études Andines 43 (2): 285-310.

Ventura, B. \& Becerra, F. 2018. Los valles orientales de Salta (Argentina) durante el dominio inca y la colonia temprana. Territorialidad, diversidad poblacional, interacción e intercambio con la Puna de Jujuy. Una mirada desde la arqueología y la etnohistoria. In Interpretando huellas. Arqueología, etnohistoria y etnografía de los Andes y sus Tierras Bajas, M. Muñoz, ed., pp. 327-347. Cochabamba: Grupo Editorial Kipus.

Villar, L., Pezzutti, N., Segal, S., Barbieri, M., Ghiara, M. \& STAnzione, D. 1997. El intrusivo alnöítico de Los Alisos, sierras subandinas, Departamento Iruya, Provincia de Salta, Argentina. Revista de la Asociación Geológica Argentina 52 (4): 459-468.

Von Rosen, E. 1957 [1919]. Un mundo que se va: exploraciones $y$ aventuras entre las altas cumbres de la cordillera de los Andes. Tucumán: Fundación Miguel Lillo-Universidad Nacional de Tucumán-Instituto Miguel Lillo.

Westfall, C., Belmar, M. \& González, C. 2010. Aplicación geo-arqueológica de microscopía electrónica y microsonda en piezas metalográficas y líticas del cementerio Regimiento Chorrillos de Calama. Actas del XVII Congreso Nacional de Arqueología Chilena, vol. 2, pp. 835-846. Santiago: Sociedad Chilena de Arqueología. 